\title{
Brockarchaeota, a novel archaeal lineage capable of methylotrophy
}

\section{Valerie De Anda}

University of Texas at Austin https://orcid.org/0000-0001-9775-0737

\section{Lin-Xing Chen}

University of California, Berkeley

\section{Nina Dombrowski}

NIOZ, Royal Netherlands Institute for Sea Research, Department of Marine Microbiology and

Biogeochemistry, and Utrecht University, P.O. Box 59, NL-1790 AB Den Burg https://orcid.org/0000-00031917-2577

\section{Zhengshuang Hua}

Sun Yat-sen University https://orcid.org/0000-0002-2405-4228

\section{Hong-Chen Jiang}

China University of Geosciences

\section{Jillian Banfield}

University of California, Berkeley https://orcid.org/0000-0001-8203-8771

\section{Wen-Jun Li}

Sun Yat-Sen University https://orcid.org/0000-0002-1233-736X

\section{Brett Baker ( $\square$ acidophile@gmail.com )}

The University of Texas at Austin https://orcid.org/0000-0002-5971-1021

\section{Article}

Keywords: Brockarchaeota, archaeal lineage, anaerobic methylotrophy

Posted Date: July 14th, 2020

DOI: https://doi.org/10.21203/rs.3.rs-39998/v1

License: (a) (i) This work is licensed under a Creative Commons Attribution 4.0 International License. Read Full License

Version of Record: A version of this preprint was published at Nature Communications on April 23rd, 2021. See the published version at https://doi.org/10.1038/s41467-021-22736-6. 


\title{
Brockarchaeota, a novel archaeal lineage capable of methylotrophy
}

\author{
Valerie De Anda ${ }^{1}$, Lin-Xing Chen ${ }^{2}$, Nina Dombrowski ${ }^{1,3}$, Zheng-Shuang Hua ${ }^{4,5}$, Hong- \\ ${ }^{1}$ Dept. of Marine Sciences, Marine Science Institute, University of Texas Austin \\ ${ }^{2}$ Department of Earth and Planetary Sciences, University of California, Berkeley, CA 94720, USA \\ ${ }^{3}$ NIOZ, Royal Netherlands Institute for Sea Research, Department of Marine Microbiology and \\ 10 Biogeochemistry, and Utrecht University, Den Burg, Netherlands \\ ${ }^{4}$ State Key Laboratory of Biocontrol and Guangdong Key Laboratory of Plant Resources, School of Life Sciences, \\ Sun Yat-Sen University, Guangzhou 510275, People's Republic of China \\ ${ }^{5}$ Department of Biological Sciences, Dartmouth College, Hanover, NH, 03755, USA \\ ${ }^{6}$ State Key Laboratory of Biogeology and Environmental Geology, China University of Geosciences, Wuhan \\ 15 430074, People's Republic of China \\ ${ }^{7}$ Department of Environmental Science, Policy and Management, University of California, Berkeley, CA 94720, \\ USA \\ ${ }^{8}$ Southern Marine Science and Engineering Guangdong Laboratory (Zhuhai), Zhuhai 519000, People's Republic of \\ China \\ $20 *$ Corresponding authors emails acidophile@gmail.com and liwenjun3@mail.sysu.edu.cn
}

Single carbon (C1) compounds such as methanol, methylamines and formaldehyde are ubiquitous in nature and they are large components of the carbon cycle. In anoxic 25 environments C1-utilizing microbes (methylotrophs) play an important role in controlling global carbon degradation. Currently described anaerobic methylotrophs are limited to methanogenic archaea, acetogenic bacteria, and sulfate-reducing bacteria. Here, we report the first archaeal lineage outside of methanogenic taxa that are capable of anaerobic methylotrophy. Phylogenetic analyses suggest these archaea form a new phylum within the

30 TACK superphylum, which we propose be named Brockarchaeota. A survey revealed Brockarchaeota are globally distributed in geothermal springs. Metabolic inference from 15 metagenome-assembled genomes from hot springs and deep-sea sediments indicates that Brockarchaeota are strict anaerobes. They contain a variety $\mathrm{C} 1$ metabolisms including the methanol and trimethylamine methyltransferases system, the ribulose bisphosphate 35 pathway coupled with the non-oxidative pentoses phosphate pathway, and reductive glycine pathway. Brockarchaeota have an incomplete methyl-branch of the Wood-Ljungdahl pathway probably used for formaldehyde oxidation, since they lack several core genes involved in methanogenesis including methyl-CoM reductases. Brockarchaeota also appear to play an important role in the breakdown of plant-derived polysaccharides, especially cellulose, starch and xylan. Their broad distribution and their capacity to use both $\mathrm{C} 1$ compounds and complex polysaccharides via anaerobic metabolism suggest that the Brockarchaeota occupy previously overlooked roles in carbon cycling.

\section{Introduction}

Methylotrophs are organisms that are capable of using single-carbon $(\mathrm{C} 1)$ compounds as a source of energy and carbon ${ }^{1,2}$. C1 compounds, such as methanol and methylamines, are derived from a variety of sources such as phytoplankton, plants, and the decay of organic matter ${ }^{3-5}$. As a result, they are ubiquitous in oceans and atmosphere and are important components of the global carbon and nitrogen cycles ${ }^{4}$. In oxic environments, $\mathrm{C} 1$ compounds are generally converted to formaldehyde by methanol dehydrogenases $(\mathrm{MDH})$ found in aerobic methylotrophs ${ }^{3,4}$. In anoxic 
settings, $\mathrm{C} 1$ compounds are used as substrate for methylotrophic-methanogenesis ${ }^{6-9}$ and sulfate reduction ${ }^{10}$. Anaerobic methylotrophs utilize the methyltransferase system (MT) to break and transfer the methyl residue of $\mathrm{C} 1$ compounds to coenzyme $\mathrm{M}$ (in the case of methanogens) or tetrahydrofolate (in acetogens and sulfate reducers) ${ }^{6-10}$ and couple this reaction to the WoodLjungdahl pathway (WLP) for energy conservation. Methylotrophic archaea include methanogenic orders (in Euryarchaeota): Methanosarcinales, Methanobacteriales, Methanomassiliicoccales and the recently discovered uncultured methylotrophic phylum, Verstraetearchaeota ${ }^{6}$. Methylotrophy has not been described in archaeal lineages outside of these

60 methanogenic groups. Also, our understanding of $\mathrm{C} 1$-utilization is limited, as there is increasing evidence that suggests $\mathrm{C} 1$ compounds serve as energetic substrates to fuel non-methanogenic heterotrophic communities in the deep subseafloor ${ }^{11}$ and marine sediments ${ }^{13}$. However, little is known about the microorganisms or pathways mediating this process ${ }^{4}$. Here we characterize a new globally distributed archaeal lineages with metabolic pathways for $\mathrm{C} 1$ utilization from hot springs and the deep ocean.

\section{Results}

\section{Genomic reconstruction}

70 Metagenomic sequencing, assembly, and binning of sediments from seven terrestrial hot springs in Tibet (up to $70^{\circ} \mathrm{C}$ ) and Tengchong Yunnan, China (up to $86^{\circ} \mathrm{C}$ ) and deep sea hydrothermallyheated Guaymas Basin (GB) sediments $\left(10-34^{\circ} \mathrm{C}\right)$ resulted in the reconstruction of 15 draft metagenome-assembled genomes (MAGs) estimated to be $67-92 \%$ complete (Table 1). These MAGs range from 0.94 to $2.90 \mathrm{Mbp}$ (average $1.85 \mathrm{Mbp}$ ) (Table 1). The two MAGs from GB

75 (B48_G17 and B27_G9, temperature 33.6 and $10.4^{\circ} \mathrm{C}$ respectively) were originally designated as "CP5" in a prior study". Although the GB genomes were obtained from lower temperatures these sediments experience increases in temperature due to hydrothermal circulation ${ }^{12}$. Thus, the organisms from which these genomes were derived likely prefer hot geothermal ecosystems, and anoxic conditions (Supporting Table 1).

\section{Phylogeny and distribution in nature}

Phylogenetic analyses of these MAGs based on a concatenated alignment of 37 conserved proteins (see Methods), revealed they form a distinct group within the TACK superphylum, basal to Aigarchaeota and Thaumarchaeota (Figure 1). A comparison of average amino acid identities

85 (AAI) across 250 available TACK genomes (Supporting Table 2) including the recently discovered phylum Marsarchaeota ${ }^{13}$, revealed that Brockarchaeota are distinct from neighboring phyla (Geoarchaeota, Aigarchaeota and Thaumarchaeota) and share up to 99\% genome-wide nucleotide similarity to one another (Supporting Figure 1 and Supporting Table 3). The two GB MAGs (B48_G17 and B27_G9) are distinct from the hot springs at the AAI

90 level $(<50 \%$ similar to each other), and $<45 \%$ AAI to members of Geoarchaeota, Thaumarchaeota and Aigarchaeota, which is consistent with their phylogenetic placement. Phylogeny of $16 \mathrm{~S}$ rRNA genes also indicated that Brockarchaeota do not fall within any described archaeal phyla (Figure 2A), with $<78 \%$ similarity to other TACK members. Together, these results support the classification of these MAGs as a new phylum. We propose that the phylum be

95 named "Brockarchaeota", after Thomas Brock, an American microbiologist known for his groundbreaking research in hot springs microbiology. 
Table 1. General information on the fifteen novel Brockarchaeota MAGs.

\begin{tabular}{|c|c|c|c|c|c|c|c|c|c|c|}
\hline Genome & Origin & $\begin{array}{c}\text { Size } \\
(\mathrm{Mb} \\
)^{\prime}\end{array}$ & $\begin{array}{l}\text { Scaffolds } \\
\text { (number) }\end{array}$ & $\begin{array}{c}\text { Protein } \\
\text { coding genes } \\
\text { (number) }\end{array}$ & $\begin{array}{l}\text { Predicted } \\
\text { size } \\
(\mathrm{Mb}) \\
\end{array}$ & $\begin{array}{l}\text { GC } \\
(\%)\end{array}$ & $\begin{array}{l}\text { Compl. } \\
\text { (\%) }\end{array}$ & $\begin{array}{l}\text { Red. } \\
(\%)\end{array}$ & $\begin{array}{l}\text { Largest } \\
\text { scaffold } \\
(\mathrm{Mb})\end{array}$ & $\begin{array}{l}\text { Strain } \\
\text { hetero }\end{array}$ \\
\hline B48_G17 & $\begin{array}{l}\text { Deep sea sediment }(12-15 \mathrm{~cm}, 33.6 \\
\left.{ }^{\circ} \mathrm{C}\right) \text { Vent } 2 \text { in Dombrowski et al } \\
2018\end{array}$ & 0.79 & 113 & 925 & 0.94 & 51 & 84 & 4.05 & 49,468 & 14.29 \\
\hline B27_G9 & $\begin{array}{l}\text { Deep sea sediment }(0-3 \mathrm{~cm}, 10.4 \\
\left.{ }^{\circ} \mathrm{C}\right) \text { Vent } 2 \text { in Dombrowski et al } \\
2018\end{array}$ & 0.99 & 223 & 1341 & 1.48 & 42 & 67 & 1.46 & 17,768 & 0 \\
\hline DRTY7 & $\begin{array}{c}\text { Hot spring sediment } \\
\text { DiReTiYanQu-7 (collected in Jan, } \\
\text { 2016) in Tengchong county, } \\
\text { Yunnan, China }\left(55.8^{\circ} \mathrm{C}\right)\end{array}$ & 0.83 & 133 & 910 & 0.99 & 34 & 84 & 1.94 & 23,553 & 0 \\
\hline QC4_43 & $\begin{array}{c}\text { Hot spring QuCai village, Tibet, } \\
\text { China }\left(69.5^{\circ} \mathrm{C}\right)\end{array}$ & 1.18 & 36 & 1332 & 1.48 & 42 & 80 & 2.43 & 135,049 & 0 \\
\hline QC4_48 & $\begin{array}{l}\text { Hot spring QuCai village, Tibet, } \\
\text { China }\left(69.5^{\circ} \mathrm{C}\right)\end{array}$ & 1.67 & 87 & 1807 & 1.99 & 48 & 84 & 9.06 & 78,542 & 12.5 \\
\hline GD2_1 & $\begin{array}{c}\text { Hot spring GuDui geothermal area, } \\
\text { Tibet, China }\left(61.8^{\circ} \mathrm{C}\right)\end{array}$ & 2.14 & 156 & 2326 & 2.40 & 48 & 89 & 1.94 & 82,330 & 0 \\
\hline QZM_A2 & $\begin{array}{c}\text { Hot spring QuZhuoMu village, } \\
\text { Tibet, China }\left(63.1^{\circ} \mathrm{C}\right)\end{array}$ & 2.19 & 173 & 2447 & 2.67 & 48 & 82 & 1.94 & 96,973 & 0 \\
\hline QZM_A3 & $\begin{array}{l}\text { Hot spring QuZhuoMu village, } \\
\text { Tibet, China }\left(62.9^{\circ} \mathrm{C}\right)\end{array}$ & 1.96 & 218 & 2281 & 2.51 & 47 & 78 & 5.83 & 70,810 & 0 \\
\hline DRTY-1.18 & $\begin{array}{c}\text { Hot spring sediment } \\
\text { DiReTiYanQu-1 (collected in } \\
\text { May, 2017) in Tengchong county, } \\
\text { Yunnan, China }\left(67^{\circ} \mathrm{C}\right)\end{array}$ & 1.62 & 55 & 1582 & 1.84 & 34 & 88 & 1.94 & 117,455 & 0 \\
\hline $\begin{array}{l}\text { DRTY- } \\
6.200\end{array}$ & $\begin{array}{c}\text { Hot spring sediment } \\
\text { DiReTiYanQu-6 (collected in } \\
\text { May, 2017) in Tengchong county, } \\
\text { Yunnan, China }\left(60^{\circ} \mathrm{C}\right)\end{array}$ & 0.55 & 129 & 663 & 0.96 & 34 & 57 & 0 & 11,975 & 0 \\
\hline DRTY-6.80 & $\begin{array}{c}\text { Hot spring sediment } \\
\text { DiReTiYanQu-6 (collected in } \\
\text { May, 2017) in Tengchong county, } \\
\text { Yunnan, China }\left(60^{\circ} \mathrm{C}\right)\end{array}$ & 2.32 & 55 & 2264 & 2.67 & 47 & 87 & 0 & 266,602 & 0 \\
\hline DRTY-7.37 & $\begin{array}{l}\text { Hot spring sediment } \\
\text { DiReTiYanQu-7 (collected in Jan, } \\
\text { 2016) in Tengchong county, } \\
\text { Yunnan, China }\left(55.8^{\circ} \mathrm{C}\right)\end{array}$ & 1.03 & 189 & 1131 & 1.18 & 35 & 87 & 0.97 & 23,662 & 0 \\
\hline JZ-1.89 & $\begin{array}{c}\text { Hot spring sediment Jinze-1 } \\
\text { (collected in May, 2017) in } \\
\text { Tengchong county, Yunnan, China } \\
\left(86.5^{\circ} \mathrm{C}\right)\end{array}$ & 1.48 & 88 & 1680 & 2.90 & 41 & 51 & 0.97 & 147,883 & 0 \\
\hline JZ-2.136 & $\begin{array}{c}\text { Hot spring sediment Jinze-2 } \\
\text { (collected in May, 2017) in } \\
\text { Tengchong county, Yunnan, China } \\
\left(63^{\circ} \mathrm{C}\right)\end{array}$ & 2.04 & 215 & 2234 & 2.43 & 58 & 84 & 0.97 & 40,656 & 0 \\
\hline JZ-2.4 & $\begin{array}{c}\text { Hot spring sediment Jinze-2 } \\
\text { (collected in Jan, 2016) in } \\
\text { Tengchong county, Yunnan, China } \\
\left(75^{\circ} \mathrm{C}\right)\end{array}$ & 1.30 & 61 & 1412 & 1.41 & 40 & 92 & 0 & 130,091 & 0 \\
\hline
\end{tabular}

Genome ID, origin, number of scaffolds, number of protein-coding genes, guanine-cytosine (GC) content, estimated completeness (Compl.), estimated gene redundancy (Red.), and strain heterogeneity (Strain hetero.) are shown. 


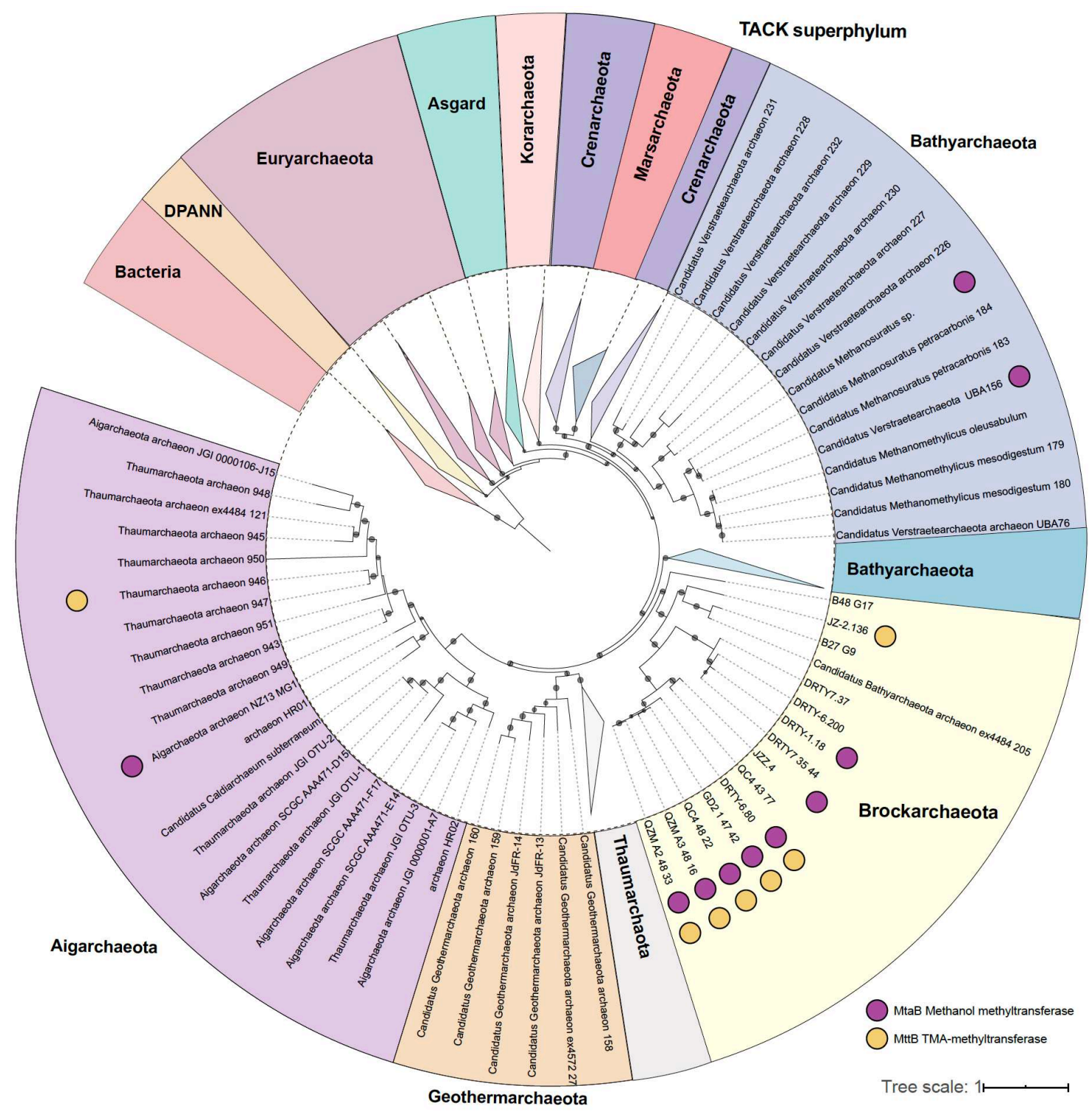

110 Figure 1. Comparison of phylogeny and distribution of methyltransferase system in Brockarchaeota and other members of TACK superphylum. Phylogeny generated using iqtreev1.6.1 using a concatenation of 37 conserved single-copy protein-coding genes described in ref $^{14}$. Bootstrap values were calculated using non-parametric bootstrapping with 100 replicates (represented by gray circles, only bootstrap $>70$ are shown). The presence methanol methyltransferase MtaB (PF12176) and trimethylamine methyltransferase

$115 \mathrm{MttB}$ (PF06253) are shown in the outer circles. The annotation was conducted with MEBS ${ }^{15}$ details can found in Supporting Table 5.

Interestingly, only three $16 \mathrm{~S}$ rRNA gene sequences with similarity (92-96\%) to Brockarchaeota sequences have been described in PCR-based surveys, highlighting the inherent 120 bias for primer choice in diversity studies. Therefore, we searched publicly available metagenomic databases to examine the geographic distribution of this phylum. Notably, we almost exclusively found 16S rRNA gene sequences related to Brockarchaeota in sequence data generated from other hot springs from around the world (China, USA, South Africa; Figure 2A), revealing Brockarchaeota are globally distributed in hot springs (Figure 2B). Three sequences, 125 which cluster together, were recovered from lake sediments in Rwanda and the Gulf of Boni in 
Indonesia $\left(28^{\circ} \mathrm{C}\right)$ (see Supporting Table 4), suggesting that some Brockarchaeota are mesophilic as well.

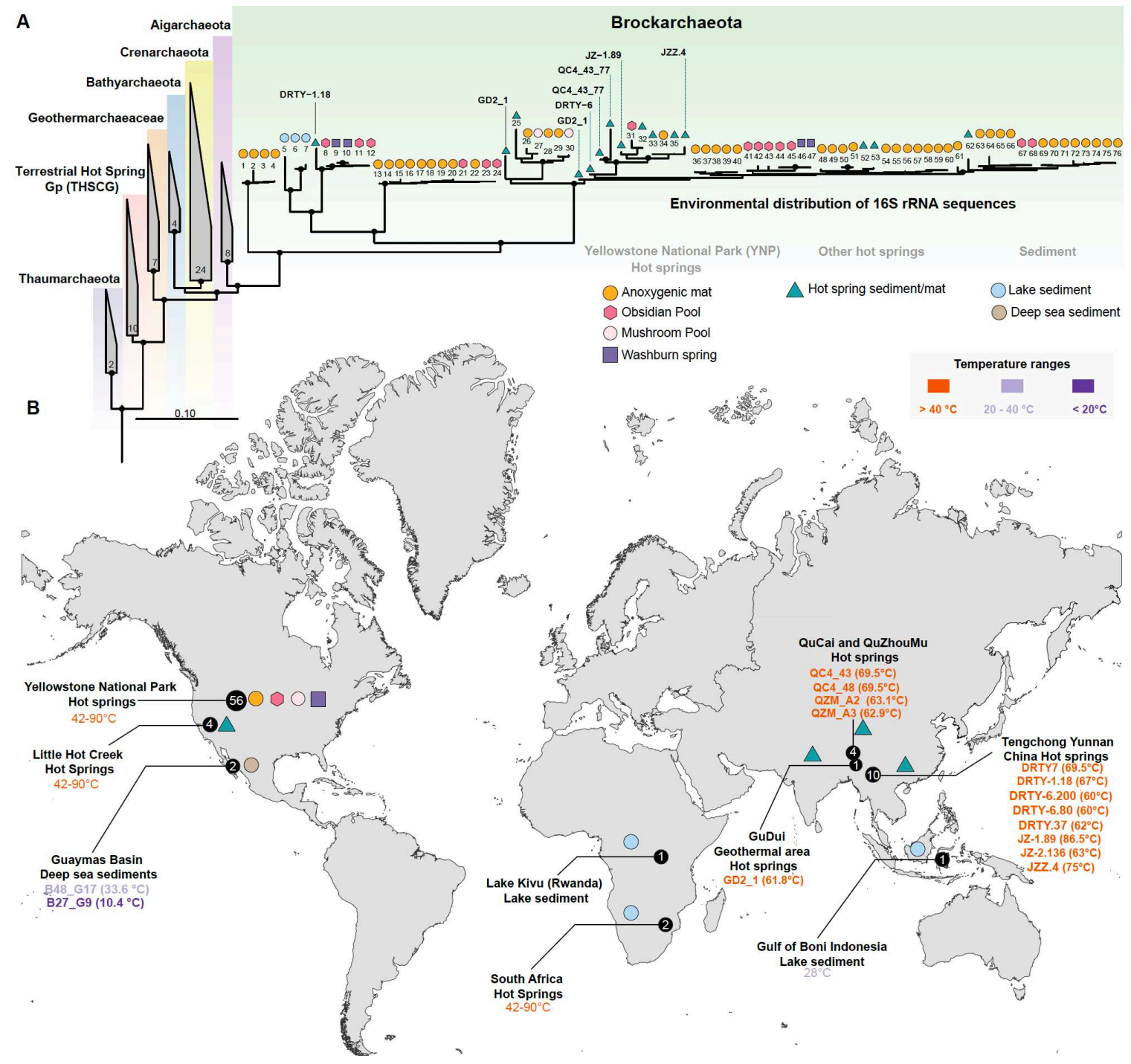

Figure 2. Location of samples from which Brockarchaeota genomes and 16S rRNA gene sequences have been recovered. (A) $16 \mathrm{~S}$ rRNA gene tree of sequences derived from metagenomic and rRNA-based diversity surveys (NCBI accessions EU924237, KX213943, and KX213897). The eight complete 16S rRNA gene sequences of Brockarchaeota genomes described in this study are shown in their respective names. Black circles in the tree represent 100 bootstrap values using RAxML with 100 replicates. Environmental information of each sequence is shown was obtained from Integrated Microbial Genomes and Microbiomes database. The number of the sequences and the corresponding metadata are described in Supporting Table 4. (B) Geographic localization from which Brockarchaeota sequences where obtained. The size of the circle corresponds to the total number of Brockarchaeota-related sequences in each geographic location. The specific MAGs obtained in this study are shown in the map according to their temperature range. 


\section{Utilization of $\mathrm{C} 1$ compounds and central carbon metabolism}

To begin to understand the metabolism of the Brockarchaeota we compared the predicted proteins encoded by these genomes with a variety of functional databases (see Methods). This revealed a

145 unique type of anaerobic methylotrophic metabolism. They contain the methyltransferase system (MT), that has been shown to be essential for anaerobic methylotrophy ${ }^{16}$ and is composed of three components. The first is a methyltransferase involved in breaking the $\mathrm{C}-\mathrm{O}$ bond of specific methylated compounds (MtaB for methanol, $\mathrm{MtmB}$ for monomethylamine, MtbB for dimethylamine, and MttB for trimethylamine). The second is the transferring methyl residue to

150 the second component, a corrinoid protein (Figure 3). The third is a methylated methylaminespecific corrinoid protein (MtaA for methanol, MtbA for methylamines), that is involved in transferring the methyl-group from the corrinoid protein to coenzyme $\mathrm{M}$ in methanogens, or tetrahydrofolate in acetogens. Brockarchaeota from hot springs encode proteins predicted to be methanol-CoM methyltransferases (MtaB) and trimethylamine-corrinoid protein 155 methyltransferase (MttB) for the utilization of methanol and trimethylamine (TMA), respectively. Brockarchaeota also encode a putative B12-binding corrinoid protein, that once methylated, can act as a substrate for the third component of the MT system that is lacking in Brockarchaeota genomes. Another undescribed protein may be involved in the transfer of the methylated compound from the corrinoid protein to unknown methyl carrier (See details in

160 Supporting Table 5). The distribution of methyltransferases among a current set of TACK superphylum genomes indicate methanol-MT system is unique feature of Brockarchaeota within the TACK superphylum (Figure 1). Prior to this, methanol-utilization is currently thought to be limited to Euryarchaeota and TACK (Verstraetaearchaeota ${ }^{6}$, Korarchaeota $^{17}$ ) archaea and some bacteria (Firmicutes and Deltaproteobacteria) ${ }^{18}$.

165 To our knowledge, no methylotrophic members of the archaea domain have been described outside methanogenic groups (Euryarchaeota and Verstraetearchaeota). Despite the presence of MT system, unlike phylogenetically related archaea such as Verstraetearchaeota ${ }^{6}$; Brockarchaeota genomes do not possess the common core marker genes specific to methanogenesis including Methyl-coenzyme M reductase (MCR) (Figure 4 and Supporting Table 6). To ensure that the MCRs

170 are not missing due to incomplete MAGs, we searched metagenomic datasets from each of communities from which they were obtained for $m c r$ genes belonging to Brockarchaeota and did not find any (See supporting Table 7 and Supporting Discussion). In addition to lacking MCR genes, they encode lack a complete Wood-Ljungdahl pathway essential for substrate utilization an energy conservation in methanogenic archaea. Furthermore, Brockarchaeota lack a methanol

175 dehydrogenase $(\mathrm{MDH})$ system that has been recently described in deep-sea sulfate reducing bacteria encoding methyltransferases ${ }^{10}$.

The lack of previously described anaerobic methylotrophic pathways, raises the question of how $\mathrm{C} 1$ compounds are assimilated by Brockarchaeota, or if they are being used for energy conservation or biosynthetic purposes. Most of the Brockarchaeota encode all four enzymes of the 180 non-oxidative pentoses phosphate pathway (NOPPP) and both key enzymes of the ribulose monophosphate pathway (RuMP) including 3-hexulose-6-phosphate synthases (HPS) and 6phospho-3-hexuloisomerases (PHI) (Supporting Figure 2). The presence of complete NOPPP, RuMP and reductive glycine pathway (rGlyP) suggests that these compounds are being assimilated into pyruvate, which can be converted to acetate for ATP formation at substrate-level phosphorylation.

The RuMP pathway was originally described in methylotrophic bacteria, which use C1 compounds as a sole source of carbon and energy, however, it is now recognized as a widespread pathway for formaldehyde fixation and detoxification ${ }^{19}$. Formaldehyde was an essential building block to synthesize sugars on early Earth and is ubiquitous in nature, produced through the degradation of compounds containing methyl- or methoxyl-groups, e.g., lignin and pectin ${ }^{20}$. In geothermally environments autotrophic microbes are thought to produce formaldehyde from a 
variety of $\mathrm{C} 1$ compounds (i.e. $\left.\mathrm{CH}_{4}, \mathrm{CO}_{2}, \mathrm{CO}\right)^{21}$. The RuMP pathway functions as an efficient system for trapping free formaldehyde at relatively low concentrations. The presence of HPS and PHI in Brockarchaeota suggests that formaldehyde can be fixed and detoxified via the RuMP pathway. Furthermore, formaldehyde can potentially be oxidized to $\mathrm{CO}_{2}$ or formate due to the presence of genes coding tungsten-dependent aldehyde ferredoxin oxidoreductase (AFOR). However, this enzyme can oxidize a wide range of different aldehydes derived from organic carbon degradation (e.g., of peptides) and may also be involved in electron transport reactions ${ }^{22}$. The presence of potential oxidative and assimilative formaldehyde pathways in Brockarchaeota may be an advantage for their survival in hot springs and hydrothermal deep-sea environments

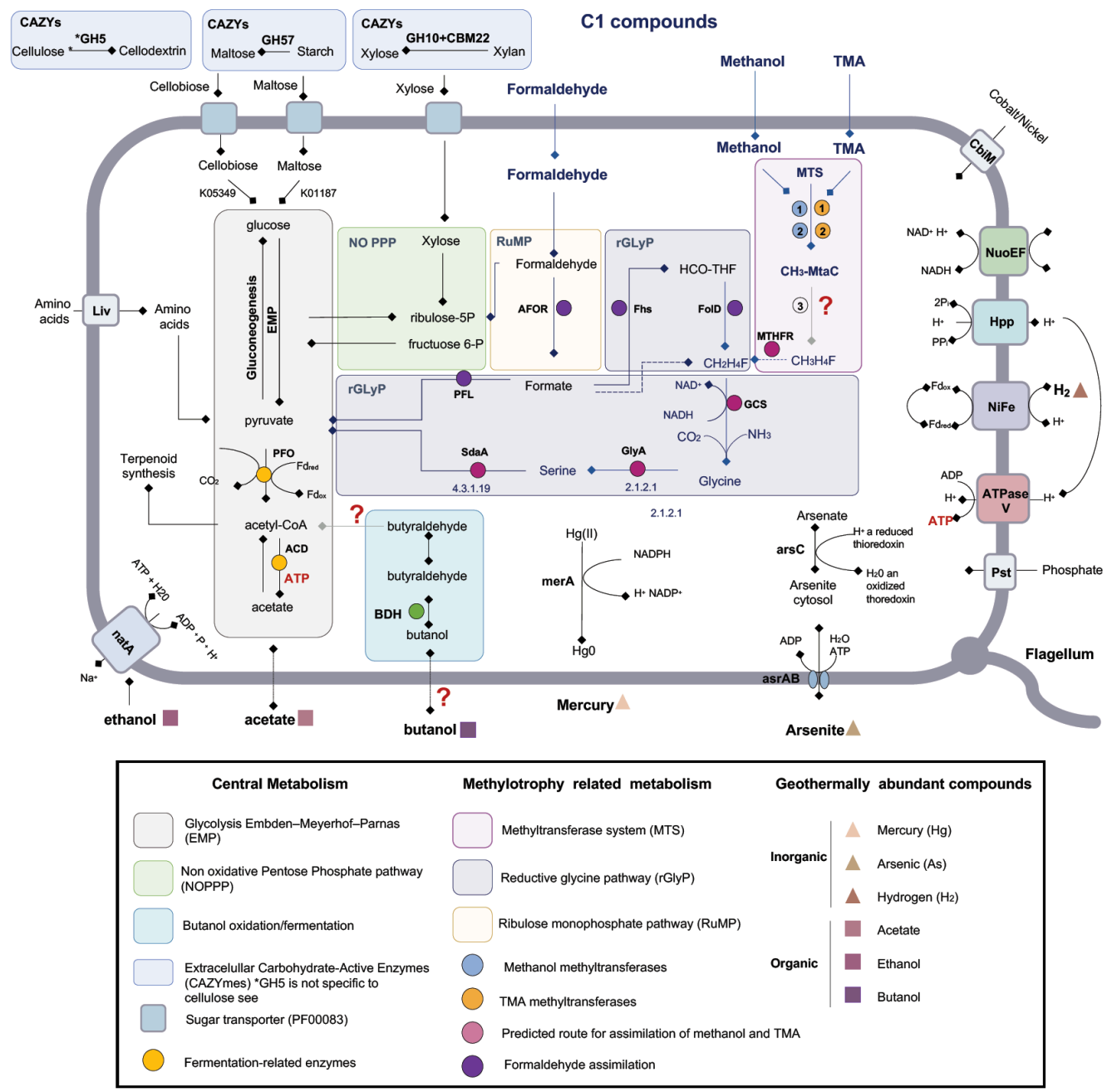

Figure 3. Overview of potential metabolic capabilities of Brockarchaeota phylum. Brockarchaota is predicted to produce ATP by substrate-level phosphorylation by fermentation of complex organic 205 compounds, pyruvate and acetate as carbon and energy sources via glycolysis (EMP) by the concerted action of PFO and ACD, that might represent the major energy-conserving reaction in this lineage. Formaldehyde can be fixed and detoxified via the ribulose monophosphate (RuMP) pathway and enter to central metabolism or be oxidized to formate by AFOR which can enter to the rGLyP pathway via FhS and FolD action C1 compounds such as methanol and TMA can be assimilated via MT system and MTHFR, generating glycine, 210 and following a linear route to central metabolism through rGLyP by subsequent conversion of serine and pyruvate. Geothermally abundant compounds are showed in colored circles Each shaded pathway can be seen in more detail in Supporting Discussion. Dashed arrow indicates that spontaneous condensation of 
formaldehyde with THF occur but is a very minor contribution to assimilation fluxes according to ref $^{36}$. For full names and copy numbers of the genes in number see Supporting Discussion. Abbreviations. Pathways: Embden-Meyerhof-Parnas (EMP), Non-Oxidative Pentoses Phosphate Pathway (NOPPP), ribulose monophosphate (RuMP), reductive glycine pathway (rGlyP). Enzymes: pyruvate ferredoxin oxidoreductase (PFO), and acetate-CoA ligase (ADP-forming) (ACD), pyruvate formate lyase (PFL), tungsten-dependent aldehyde ferredoxin oxidoreductase (AFOR), tetrahydrofolate (THF) ligase (FhS), methenyl-THF cyclohydrolase/methylene-THF dehydrogenase (FolD), methylene-THF reductase MTHFR 220 (MetFmethyltransferase system (MT), glyA, serine hydroxymethyltransferase (GlyA), serine deaminase (SdaA), glycine-cleavage system (GCS). Compounds: Single carbon compounds (C1), trimethylamine (TMA). The three main components of the methyltransferase system are explained in the main text.

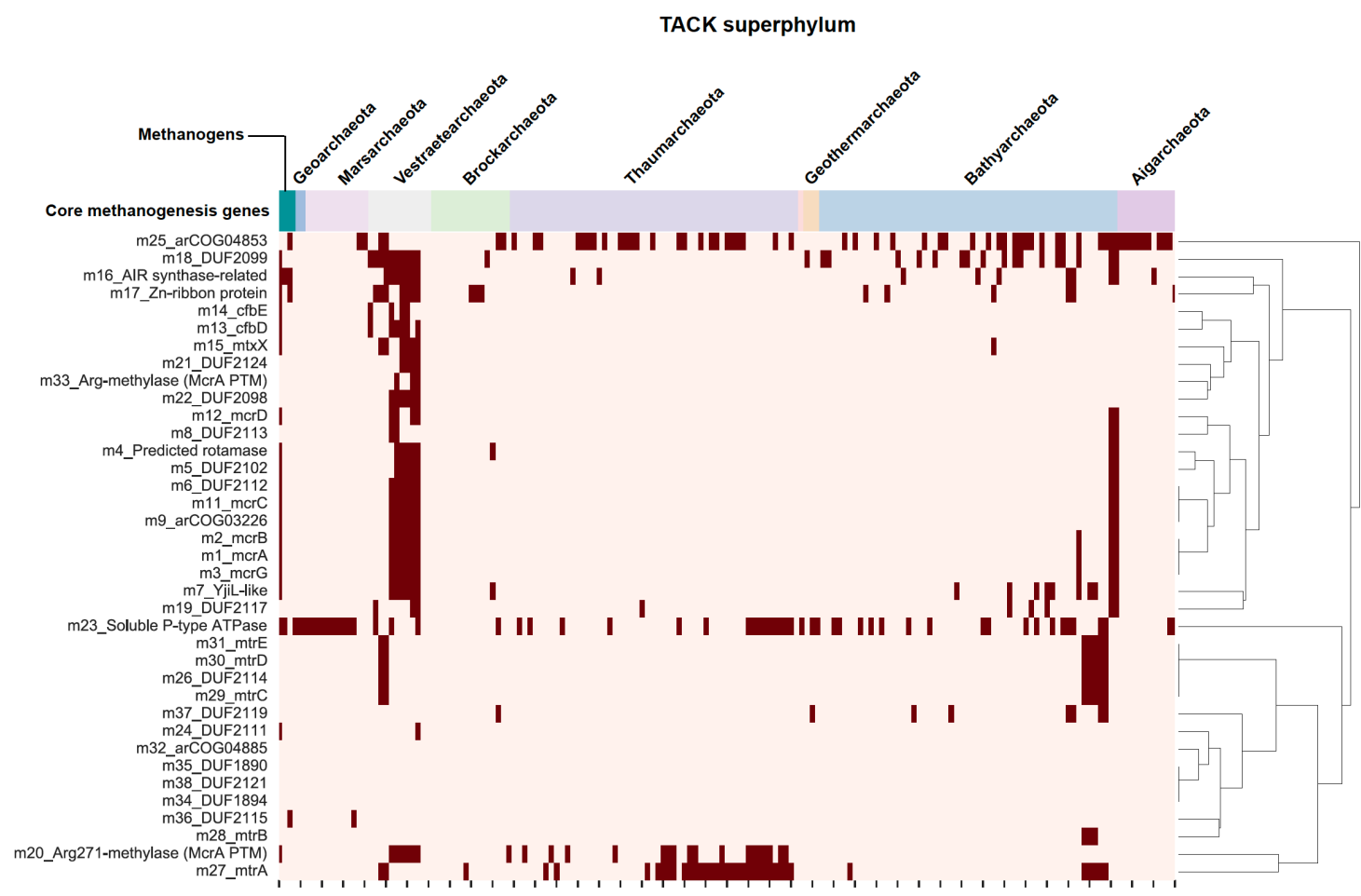

225 Figure 4. Presence and absence profile of common core marker genes specific to methanogenesis across the TACK superphylum. Genes include those associated to methanogenesis, anaerobic methanotrophic and short-chain alkane-oxidizing archaea described in Greening et al. $2016^{34}$ (see Supporting Table 6 for specific details of the genomes used in this study and the specific marker genes named with $\mathrm{m}$ followed with a number in the figure). For comparison purposes the following known methanogenic archaea were included: Candidatus Methanoplasma termitum that lacks the entire pathway for $\mathrm{CO}_{2}$ reduction to methyl coenzyme $\mathrm{M}$ and produces methane by hydrogen-dependent reduction of methanol or methylamine (Methanomassiliicoccales), Methanosphaera stadtmanae (Methanobacteriales) that can generate methane only by the reduction of methanol with $\mathrm{H}_{2}$ and is dependent on acetate as a carbon source, and Methanosarcina acetivorans (Methanosarcinales).

Some Brockarchaeota genomes code tetrahydrofolate (THF) ligase (fhs), methenyl-THF cyclohydrolase/methylene-THF dehydrogenase ( $f o l D)$, and methylene-THF reductase MTHFR $(m e t F)$, which are key enzymes of the methyl-branch of the Wood-Ljungdahl pathway (Supporting 240 Table 5). Nonetheless, given the absence of key carbonyl branch genes, Brockarchaeota appears to be incapable of assimilating C1 compounds via the WL pathway used by other methylotrophic archaea. Yet, the presence of genes for the rGLYp pahtway ( $g c v P$, glycine dehydrogenase; $g c v T$, aminomethyltransferase; $g c v H$, lipoate-binding protein; $l p d$, dihydrolipoyl dehydrogenase; $g l y A$, 
serine hydroxymethyltransferase; and $s d a A$, serine deaminase) in Brockarchaeota genomes, offers another alternative route for assimilation of $\mathrm{C} 1$ compounds not yet described in methylotrophic archaea. Subsequent conversion of C1 compounds in Brockarchaeota can proceed via methyleneTHF derivatives, either by MTHFR or FoldD (in the case of formaldehyde) to generate $5,10-\mathrm{CH}_{2}-$ tetrahydrofolate which can be assimilated via glycine-cleavage system $(\mathrm{GCS})^{23}$, followed by the assimilation into central metabolism with the conversion of serine into pyruvate (see figure 3 ).

250 A phylogenetic analysis of alcohol dehydrogenases from hot spring genomes revealed that they encode a butanol dehydrogenase BDH (Supporting Figure 3) that catalyzes the reversible conversion of butyraldehyde to butanol. Brockarchaeota BDH's are homologues to sequences from obligately anaerobic, thermophilic bacteria that can degrade complex plant saccharides such as xylan (i.e Caldicoprobacter oshimai ${ }^{24}$ and Hungateiclostridium thermocellum ${ }^{25}$ ) or cellulose

255 (Hungateiclostridium alkalicellulosi). To investigate if Brockarchaeota can oxidize or produce butanol, we searched for genes involved in production of butanol in two model organisms; Clostridium acetobutylicum ${ }^{26,27}$ which is one of the few organisms that produces butanol as a fermentation product, and Saccharomyces cerevisiae ${ }^{28}$ involved in butanol and isopropanol production. We found that Brockarchaeota genomes lack the key enzymes involved in the

260 fermentation of pyruvate to butanol (butanal dehydrogenase, butyryl-CoA dehydrogenase, enoylCoA dehydratase, 3-hydroxyacyl-CoA dehydrogenase). However, most of the genomes code a putative aldehyde dehydrogenase that could convert butyraldehyde to butyric acid. Also, we found a putative enoyl-CoA hydratase/isomerase protein that is coded by one bin (JZ-1.89), which could be involved in further converting butyric acid to acetyl-CoA. Our results suggest an alternative 265 pathway for butanol oxidation that still remains unresolved (Supporting Figure 4).

\section{Pathways for the utilization of extracellular organic carbon}

In addition to anaerobic methylotrophy, Brockarchaeota may be able to degrade a variety of organic carbon compounds. They may utilize hexoses via Embden-Meyerhof-Parnas (EMP) 270 pathway (Supporting Figure 5) and pentoses (xylose isomerase $x y l A$ and xylulose kinase $x y l B$ ) via the isomerase pathway (Supporting Figure 2). These enzymes were previously only found in bacterial thermophiles and halophilic archaea that ferment complex compounds and degrade xylose suggesting a similar physiology in Brockarchaeota ${ }^{29}$. Once assimilated into the cell, carbon complex compounds could enter the central metabolism and be converted to acetate and $\mathrm{H}_{2}$ via

275 acetogenic fermentation. The ATP conserving step or either sugar or pyruvate fermentation to acetate could be catalyzed by acetate-CoA ligase in the hot spring genomes. Acetate can also be assimilated to acetyl-CoA by acetyl-CoA synthetase (ACS), thus acetate might be a source of carbon and energy in the absence of other substrates in hot spring Brockarchaeota. The presence of pyruvate ferredoxin oxidoreductase (PFO) that couples pyruvate oxidation to $\mathrm{H}_{2}$ production, 280 generating acetyl-CoA, could support fermentative metabolism via degradation of either acetate, pyruvate, hexoses or pentoses. Brockarchaeota genomes appear to code a wide repertoire of ATPases such as the plasma-membrane proton-efflux P-type ATPase (only present in the hot spring genomes), $\mathrm{Zn} 2+/ \mathrm{Cd}^{2+-}$ exporting ATPase (present in DRTY7.37), and finally the V/A-type $\mathrm{H}+/ \mathrm{Na}+-$ transporting ATPase (in most of the genomes). The existence of ATPase in Brockarchaeota

285 suggests that members of these genotypes have the additional ability to couple acetogenic fermentation to membrane potential generation of a transmembrane ion gradient across the membrane.

To complement their ability to degrade xylenes, Brockarchaeota also contain a relatively high number of carbohydrate-active enzymes (average of 27 CAZYmes per genome) which is 3

290 times what has been observed in other TACK archaea (Supporting Table 8 and Figure 5). Ten of the 15 Brockarchaeota genomes have genes with similarity to $\alpha$-L-fucosidase involved in the degradation of xyloglucan, which is the major component of hemicellulose in plant-cell walls ${ }^{30}$. All the hot spring genomes encode GH3 family proteins for detrital cellulose degradation, plant 
and bacterial cell wall remodeling, energy metabolism, and pathogen defense $\mathrm{e}^{31}$. The hot spring genotypes contain a wider repertoire of CAZymes than the deep-sea GB genomes. Among these are four predicted to be extracellular glycoside hydrolases, which are involved in the breakdown of high molecular-weight plant-derived polysaccharides, primarily xylanes, cellulose, and starch. Comparison of the CAZYmes across the TACK superphylum revealed that 17 extracellular enzymes, including enzymes for the degradation xylanes, are unique to Brockarchaeota (Supporting

300 Table 8). The diversity and abundance of CAZYmes in members of the TACK superphylum highlights that that despite the low number of sequenced Brockarchaeota genomes (15 described in this study) compared to Thaumarchaeota (89), the former encodes a wider arrange and in wider abundance that their phylogenetically related counterparts.

305

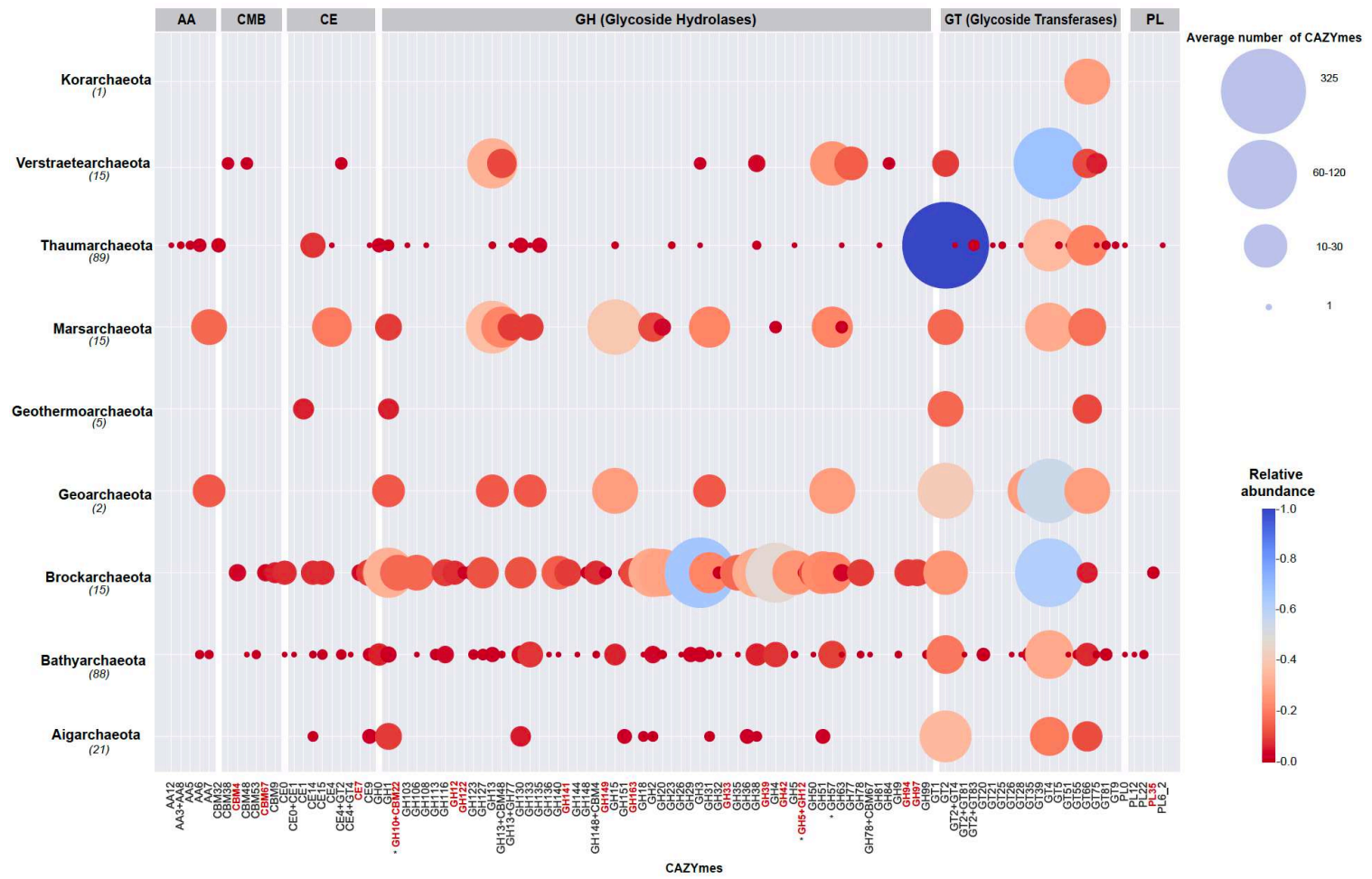

Figure 5. Annotated carbohydrate-active enzymes (CAZymes) encoded by the total number of genomes belonging to the TACK superphylum including Brockarchaeota. The total number of CAZymes per phylum was normalized by the total number of genomes described for each phylum shown

310 in parenthesis. Auxiliary activities (AA), carbohydrate-binding module (CBM), carbohydrate esterases (CE), glycoside-hydrolases (GH), glycoside transferases (GT), polysaccharide lyases (PL). Unique Brockarchaeota CAZymes are shown in red. Extracellular CAZYmes in Brockarchaeota are shown in asterisks described in Supporting Table 8 sheet 3.

\section{Hydrogen metabolism}

Hydrogen is also abundant in hydrothermal systems due to volcanic processes ${ }^{32}$. Brockarchaeota might be able to use $3 \mathrm{~b}$ [NiFe]-hydrogenases for $\mathrm{H}_{2}$ oxidation with $\mathrm{NADP}+$ or $\mathrm{NAD}(\mathrm{P})+$ as an electron acceptor ${ }^{33}$. The hot spring genomes also encode oxygen-tolerant group $3 \mathrm{~d}[\mathrm{NiFe}]-$ 320 hydrogenases, which may allow them to transfer electrons between $\mathrm{NAD}(\mathrm{P}) \mathrm{H}$ and $\mathrm{H}_{2}$ depending on the availability of electron acceptors (Supporting Figure 6). Group 3d [NiFe]-hydrogenases are abundant in metagenomes from hot springs where microbial communities are relatively stable 
despite partial pressure of oxygen fluctuations ${ }^{34}$. Group [NiFe] 3b hydrogenases may also make it possible for these archaea to reduce elemental sulfur to $\mathrm{H}_{2} \mathrm{~S}$ during fermentative growth. During 325 carbohydrate fermentation in the absence of sulfur, Group $3 \mathrm{~b}$ [NiFe]-hydrogenases might catalyze the production of $\mathrm{H}_{2}$ with $\mathrm{NADPH}$ or $\mathrm{NAD}(\mathrm{P}) \mathrm{H}$ as the electron donor. Therefore, Brockarchaeota might have the ability to reduce sulfur, using $\mathrm{H}_{2}$ or organic substrates as electron donors, which is common in hyperthermophilic archaea living in geothermally active environments ${ }^{35}$

\section{Discussion}

Brockarchaeota gene contents suggests they are facultative or obligate anaerobic fermentative organisms that produce acetate, $\mathrm{CO}_{2}$, and $\mathrm{H}_{2}$ as byproducts (see Supporting Information for details). Brockarchaeota are also able to degrade complex carbon compounds such as xylenes and perform

335 anaerobic methylotrophy. Xylenes are a major structural polysaccharide in plant cells, and is the second most abundant polysaccharide in nature, accounting for approximately one-third of all renewable organic carbon on Earth after cellulose ${ }^{37,38}$. This suggests that Brockarchaeota are key players in organic matter degradation in hot springs and deep-sea sediments. Furthermore, Brockarchaeota have unique pathways for non-methanogenic methylotrophy which has not been

340 described in the archaea domain prior to this and has not been attributed to any organism broadly distributed in nature. This gives them a unique ecological position in nature, to degrade abundant methylamines in anoxic environments (Figure 6).

The protein repertoire of GB and hot springs genomes have some important distinctions that likely reflects different anaerobic physiologies. GB genomes appear to be obligately

345 fermenting organisms that rely mostly on substrate-level phosphorylation since they lack all the complexes for the respiratory chain with exception of the ATPase. In contrast, hot spring genomes appear to have mechanisms to increase their ATP yield including the use of geothermally derived inorganic substrates as possible terminal electron acceptors such as mercury (Hg), arsenic (As) and hydrogen $\left(\mathrm{H}_{2}\right)$. Deep-sea hydrothermal vents, hot springs, and fumaroles are natural sources of $350 \mathrm{Hg}^{39}, \mathrm{H}_{2}{ }^{34}$ Arsenic $^{40}$ and sulfur ${ }^{41}$. Three hot springs Brockarchaeota genomes (DRTY735_44, DRTY-1.18 and DRTY.37) encode mercuric reductase (MerA), which detoxifies $\mathrm{Hg}$ (II) to $\mathrm{Hg}(\overline{0})^{39}$. Brockarchaeota also appear to have the genetic capacity for As-resistance including arsenate reductase (ArsC) for the reduction of arsenate to arsenite, which is present in most of the hot spring genomes, and arsenite efflux transporters ( $a s r A$ and $a s r B$ ) for cell export (Figure 3 ). The presence

355 of this energy-dependent efflux process related detoxification proteins, could also indicate that Brockarchaeota in hot springs genomes could use arsenate as terminal electron acceptor, as seen in bacteria $^{42,43}$

The discovery of Brockarchaeota genomes from sediments around the world, overlooked by conventional rRNA gene diversity approaches, highlights the need for further exploration of

360 subsurface microbial communities. The addition of these genomes to public databases, like other recently described novel archaeal lineages ${ }^{44-46}$, will enhance their detection in future environmental studies. A lack of recognition of their existence prior to this, limited our ability to fully describe sediment community structure and function. Given their broad distribution, and versatile carbon metabolism, they are likely key players in global carbon cycling. However, this first description is

365 limited to genomic characterization, thus culturing or in activity measurements are needed to confirm their physiological activities ${ }^{47}$. Overall, the description of this new phylum enhances our understanding of biodiversity of archaea and suggests they are mediating unique roles in anoxic carbon cycling. 


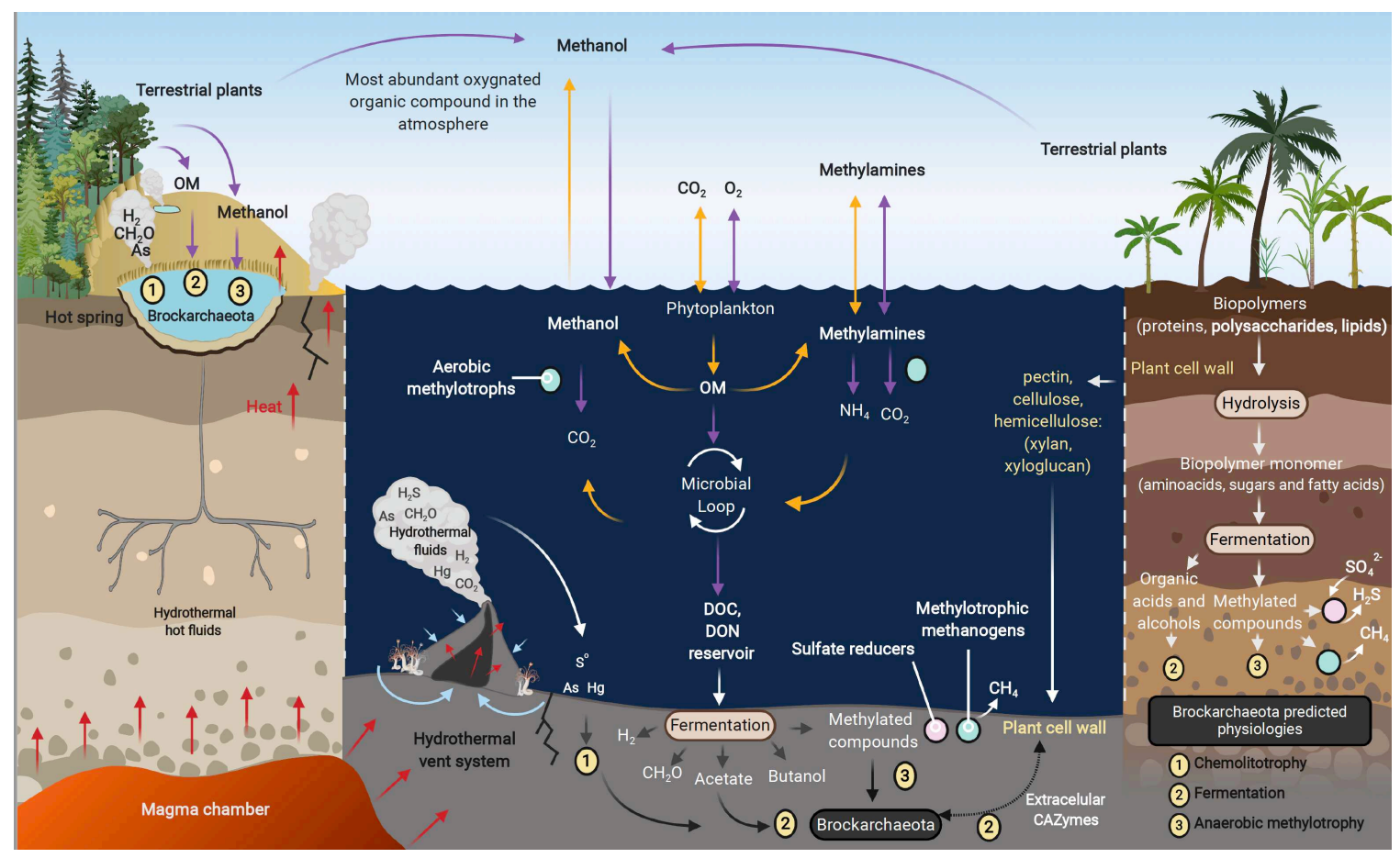

Figure 6. The role of Brockarchaeota in the anaerobic carbon cycle. Single carbon (C1)-methylated compounds, such as methanol or methylamines, are utilized biologically as carbon and energy sources in the ocean and deep-sea sediments resulting in a considerable carbon reservoir. The biodegradation of organic carbon in the water column and subsurface is a source of C1-methylated compounds. The utilization of methyl compounds as precursors in methane synthesis is confined to a small group of methylotrophic methanogens (i.e Verstraetearchaeota). The only described anaerobic methylotrophs include members of methanogenic archaea, acetogenic bacteria, and sulfate-reducing bacteria. These organisms compete for $\mathrm{C} 1$

380 compounds geochemically produced in anoxic settings. Brockarchaeota may recycle $\mathrm{C} 1$ in anoxic environments without methane formation and may be sequestered in deep sea sediments and hot springs. Orange and purple arrows represent sources and sinks, respectively. Organic Matter (OM) includes dissolved and particulate organic matter feeding the microbial loop (Adapted from Evans et al., 2019 and Zhuang et al., 2018).

\section{Methods}

Metagenomic assembly and binning. Two MAGs (B48_G17 and B27_G9) were obtained from 390 Guaymas Basin sediments (Gulf of California; $27^{\circ} \mathrm{N} 0.38 \overline{8}, 111^{\circ} \mathrm{W} 24.5 \overline{6} 0$ ) and were obtained as part of a larger study of these hydrothermal marine sediments ${ }^{12}$. Both samples were collected from the same location but G9 was sampled from $0-3 \mathrm{~cm}$ and $\mathrm{G} 17$ form $12-15 \mathrm{~cm}$ depth. The sediment cores from which these two MAGs were binned from were collected during Alvin dive 4571_4 in 2009 using polycarbonate cores (45-60 cm in length, $6.25 \mathrm{~cm}$ interior diameter), subsampled into $395 \mathrm{~cm}$ layers under $\mathrm{N}_{2}$ gas in the ship's laboratory and immediately frozen at $-80^{\circ} \mathrm{C}$. Details on the sampling site and metagenomic sequencing effort is provided in Dombrowski et al., 2018.

Briefly, total DNA from $\geq 10 \mathrm{~g}$ of sediment from each sample was extracted using the MoBio PowerMax soil kit using the manufacturer's instructions and adjusted to a final concentration of $10 \mathrm{ng} / \mu 1$ of each sample (using a total amount of $100 \mathrm{ng}$ ). Libraries for paired-end

400 Illumina (HiSeq-2500 1TB) sequencing were prepared by the Joint Genome Institute (JGI). Sequencing was performed on an Illumina HiSeq 2500 machine using the paired-end 2x125 bp run- 
type mode. All runs combined provided a total of $\sim 280$ gigabases of sequencing data. Quality control and sequence assembly was performed by JGI. For further binning, only scaffolds $\geq 2000$ bps were included.

405 Metagenomic binning was performed on individual assemblies using the binning tools ESOM, Anvi'o (v2.2.2) ${ }^{48}$ and Metabat (v1) ${ }^{49}$. For ESOM bins were extracted using getClassFasta.pl and the command -loyal 51. Anvi'o was run with default parameters and metabat was run using the following settings: --minProb 75 --minContig 2000 --minContigByCorr 2000. Results from the three different binning tools were combined using DAS Tool (version 1.0) as 410 follows: DAS_Tool.sh -i Anvio_contig_list.tsv, Metabat_contig_list.tsv, ESOM_contig_list.tsv -1 Anvio, Metabat, ESOM -c scaffolds.fasta --write_bins 1. The accuracy of the binning approach was evaluated by calculating the percentage of completeness and contamination using CheckM lineage_wf (v1.0.5).

Six additional MAGs (QC4 43, QC4 48, GD2 147 42, QZM A2, QZM A3, and

415 DRTY7) were recovered from hot springs in Tibet and Yunnan, China collected in August of 2016 in several hot springs. Sequencing was done on an Illumina HiSeq4000 (Beijing Novogene Bioinformatics Technology Co., Ltd). The Chinese MAGs were assembled using metaSPADES (version 3.10.1), with a k-mer set of " $21,33,55,77,99,127$ ". For each sample only scaffolds larger than $2500 \mathrm{bp}$ were binned using MetaBAT (v.1) with default parameters, considering both

420 tetranucleotide frequencies (TNF) and scaffold coverage information. The scaffolds from the obtained bins and the unbinned scaffolds were visualized using ESOM with a minimum length of $2500 \mathrm{bp}$ and maximum length of $5000 \mathrm{bp}$ as previously described ${ }^{50}$ and the bins were modified by removing any out-of-range scaffolds (indicated by sequence points) or adding any unbinned scaffolds using ESOM related scripts ${ }^{43}$. Also, scaffolds $\geq 1000 \mathrm{bp}$ from each sample were uploaded 425 to ggKbase (http://ggkbase.berkeley.edu/), and the bins from ESOM analyses were evaluated and modified manually at ggKbase based on GC content, coverage and taxonomic information of scaffolds.

Phylogenetic analyses. A phylogenetic tree was generated as recently described in ref $^{46}$. Briefly, 43036 conserved marker proteins were extracted using phylosift ${ }^{51}$, in a genomic dataset containing 3,549 archaeal genomes including Brockarchaeota, and 40 bacterial genomes. An alignment of the 36 individual proteins extracted from a total of 3,599 genomes was generated using MAFFT (algorithm autoselection) with a BLOSUM62 scoring and contains 4,962 characters after masking gaps present in at least $50 \%$ of the taxa. A tree was constructed withIQtree (v1.6.11) with a best

435 fit $\mathrm{LG}+\mathrm{F}+\mathrm{R} 10$ model selected using the Bayesian Information Criterion (BIC) and bootstraps are based on 1000 replicated trees. The bacterial genomes were used as an outgroup. The $16 \mathrm{~S}$ rRNA sequences were extracted from Brockarchaeota genomes using Barrnap (https://github.com/Victorian-Bioinformatics-Consortium/barrnap) and used for a 16S rRNA gene phylogeny that included sequences derived from metagenomic surveys (NCBI accession 440 EU924237, KX213943, and KX213897) and the IMG database. The rRNA phylogeny was generated using RAxML within the ARB software package (v. 2.5b). using default parameters.

Metabolic predictions. Gene predictions for individual genomes were performed using Prodigal ${ }^{52}$ (V2.6.2, default settings). Predicted genes of individual genomes were further characterized using 445 a combination of several databases: KofamKOALA ${ }^{53}$, Interproscan v5.31.70 ${ }^{54}$, HydDB ${ }^{55}$, dbCAN $2^{56}$, MEBS $^{57}$ and METABOLIC ${ }^{58}$. For KofamKOALA only hits above the predefined threshold for individual KOs were selected. Hydrogenases were extracted using the reference database described in ref ${ }^{34,55}$ where there was conflict, the protein was manually reanalyzed using BLAST against non-redundant protein database, and genomic organization and annotation was 
450 confirmed using a web-based tool Operon Mapper ${ }^{59}$. The detected hydrogenases were used to generate a phylogenetic tree as previously described in ref $^{45}$. Hits for key metabolic marker genes were verified across different databases KofamKOALA, PFAMv31 and TIGRFAMs and HydDB and were further verified using BLASTP using the NCBI web server tool. Genes encoding for carbohydrate degradation enzymes described in the Carbohydrate-Active enZYmes (CAZYmes)

455 database ${ }^{60}$ were identified by only retaining hits recovered by $\geq 2$ tools. Protein localization of the selected CAZYmes was determined with the command line version of Psort (V3.0) ${ }^{61}$ using the options -a and -terse for archaeal genomes in tabular format files. Finally, the presence of specific protein families was obtained with MEBS. The annotation was performed in a genomic dataset of 250 publicly available TACK genomes (Supporting Table 2) that were also used for the CAZYmes 460 annotation.

\section{Methyl coenzyme $M$ reductase screening.}

The mcrA gene was identified using GraftM v0.10.2 ${ }^{62}$ across metagenome assemblies where Brockarchaeota genomes from hot springs were detected ${ }^{63}$. The $m c r A$-containing scaffolds with 465 sequence length $<2.5 \mathrm{Kbp}$ were discarded since scaffolds with short length were not used during the genome binning step. The taxonomic information of the corresponding bins which contain merA genes were determined using either GTDBtk v0.3.2 ${ }^{64}$ or phylogenetic placement (as reported in Supplementary Table 9). The mcr $A B G C D$ genes were identified in metagenome assemblies from deepsea assemblies previously described in ref ${ }^{12}$ (Guay17 and Guay9; IMG genome ID 3300014887 and 4703300013103 respectively).

\section{Data availability}

The final assembled and annotated genomic sequences of Brockarchaeota from deep sea sediments (B27_G9 and B48_G17) have been deposited in NCBI under BioProject ID PRJNA362212:

475 BioSample id SAMN09215183 and SAMN09214986 respectively. Sequence data and sample information of Brockarchaeota from hot springs are available at NCBI under Bio Project ID PRJNA544494.

\section{Author contributions}

480 J.F.B, W-J.L., and B.J.B conceived the study. H.C.J. and Z.S.H performed sampling at Tibet and Yunnan hot springs. L.X.C. and N.D. reconstructed and curated the genomes. V.D.A and B.J.B. generated the phylogenetic trees. V.D.A., N.D., and B.J.B. generated the metabolic reconstructions. V.D.A and B.J.B performed the genomic comparisons and physiology inference. V.D.A. and B.J.B. wrote the manuscript with input for all the other authors.

\section{Acknowledgements}

We thank Dr. Thomas Brock for a career of transformative microbiological research and graciously allowing us to name Brockarchaeota in his honor. The work conducted by the U.S. Department of Energy Joint Genome Institute, a DOE Office of Science User Facility, is supported by the Office

490 of Science of the U.S. Department of Energy under Contract No. DE-AC02-05CH11231 provided to ND. This work was funded by a National Science Foundation DEB: Systematics and Biodiversity Sciences (grant number 1753661) provided to BJB. This work was supported by Science and Technology Infrastructure work project of China Ministry of Science and Technology (No. 2015FY110100), National Natural Science Foundation of China (Nos. 91951205 and 91751206).

495 We thank Andreas for providing the sediments from Guaymas Basin. Sampling in Guaymas Basin was supported by NSF Awards OCE-0647633 to Dr. Andreas P. Teske. 
1. Chistoserdova, L. Modularity of methylotrophy, revisited. Environ. Microbiol. 13, 26032622 (2011).

2. Chistoserdova, L. \& Kalyuzhnaya, M. G. Current Trends in Methylotrophy. Trends

$505 \quad$ Microbiol. 26, 703-714 (2018).

3. Sun, J., Mausz, M. A., Chen, Y. \& Giovannoni, S. J. Microbial trimethylamine metabolism in marine environments. Environ. Microbiol. 21, 513-520 (2018).

4. Zhuang, G. C., Peña-Montenegro, T. D., Montgomery, A., Hunter, K. S. \& Joye, S. B. Microbial metabolism of methanol and methylamine in the Gulf of Mexico: insight into

510 marine carbon and nitrogen cycling. Environ. Microbiol. 20, 4543-4554 (2018).

5. Zhuang, G.-C., Montgomery, A. \& Joye, S. B. Heterotrophic metabolism of C1 and C2 low molecular weight compounds in northern Gulf of Mexico sediments: controlling factors and implications for organic carbon degradation. Geochim. Cosmochim. Acta 247, 243-260 (2019).

515 6. Vanwonterghem, I. et al. Methylotrophic methanogenesis discovered in the archaeal phylum Verstraetearchaeota. Nat. Microbiol. 1, 1-9 (2016).

7. Lazar, C. S. et al. Genomic evidence for distinct carbon substrate preferences and ecological niches of Bathyarchaeota in estuarine sediments. Environ. Microbiol. 18, 12001211 (2016).

520 8. Zhuang, G. Methylotrophic methanogenesis and potential methylated substrates in marine sediment. (University of Bremen, 2014).

9. Richards, M. A. et al. Exploring hydrogenotrophic methanogenesis: A genome scale metabolic reconstruction of Methanococcus maripaludis. J. Bacteriol. 198, 3379-3390 (2016).

525 10. Sousa, D. Z. et al. The deep-subsurface sulfate reducer Desulfotomaculum kuznetsovii employs two methanol-degrading pathways. Nat. Commun. 9, (2018).

11. Trembath-Reichert, E. et al. Methyl-compound use and slow growth characterize microbial life in 2-km-deep subseafloor coal and shale beds. Proc. Natl. Acad. Sci. 114, E9206-E9215 (2017).

530 12. Dombrowski, N., Teske, A. P. \& Baker, B. J. Extensive metabolic versatility and redundancy in microbially diverse, dynamic Guaymas Basin hydrothermal sediments. Nat. Commun. doi:10.1038/s41467-018-07418-0.

13. Jay, Z. J. et al. Marsarchaeota are an aerobic archaeal lineage abundant in geothermal iron oxide microbial mats. Nat. Microbiol. 3, 732-740 (2018).

535 14. Darling, A. E. et al. PhyloSift: phylogenetic analysis of genomes and metagenomes. PeerJ 2, e243 (2014).

15. De Anda, V. et al. MEBS, a software platform to evaluate large (meta)genomic collections according to their metabolic machinery: unraveling the sulfur cycle Authors. Gigascience 6, 1-17 (2017).

540 16. Fricke, W. F. et al. The Genome Sequence of Methanosphaera stadtmanae Reveals Why This Human Intestinal Archaeon Is Restricted to Methanol and H 2 for Methane Formation and ATP Synthesis $\uparrow$. 188, 642-658 (2006).

17. McKay L, Dlakic M, Fields M, Jay Z, Eren M, Delmont T, Klingelsmith K, Rusch D, I. W. Co-occurring genomic capacity for anaerobic methane and dissimilatory sulfur metabolisms discovered in the Korarchaeota. Nat. Microbiol. (2019) doi:10.1038/s41564019-0362-4.

18. Muñoz-Velasco, I. et al. Methanogenesis on Early Stages of Life: Ancient but Not Primordial. Orig. Life Evol. Biosph. 1-14 (2019) doi:10.1007/s11084-018-9570-9.

19. Orita, I. et al. The ribulose monophosphate pathway substitutes for the missing pentose 
550 phosphate pathway in the archaeon Thermococcus kodakaraensis. J. Bacteriol. 188, 46984704 (2006).

20. Orita, I. et al. The archaeon Pyrococcus horikoshii possesses a bifunctional enzyme for formaldehyde fixation via the ribulose monophosphate pathway. J. Bacteriol. 187, 36363642 (2005).

555 21. Moran, J. J. et al. Formaldehyde as a carbon and electron shuttle between autotroph and heterotroph populations in acidic hydrothermal vents of Norris Geyser Basin, Yellowstone National Park. Extremophiles 20, 291-299 (2016).

22. Bräsen, C., Esser, D., Rauch, B. \& Siebers, B. Carbohydrate metabolism in archaea: Current insights into unusual enzymes and pathways and their regulation. JAMA

$560 \quad$ Ophthalmol. 132, 326-331 (2014).

23. Lokanath, N. K., Kuroishi, C., Okazaki, N. \& Kunishima, N. Purification, crystallization and preliminary crystallographic analysis of the glycine-cleavage system component Tprotein from Pyrococcus horikoshii OT3. Acta Crystallogr. Sect. D Biol. Crystallogr. 60, 1450-1452 (2004).

565 24. Yokohama, H., Wagner, I. D. \& Wiegel, J. Caldicoprobacter oshimai gen. nov., sp. nov., an anaerobic, xylanolytic, extremely thermophilic bacterium isolated from sheep faeces, and proposal of Caldicoprobacteraceae fam. nov. Int. J. Syst. Evol. Microbiol. 60, 67-71 (2010).

25. Zhang, X. et al. Petroclostridium xylanilyticum gen. Nov., sp. nov., a xylan-degrading 570 bacterium isolated from an oilfield, and reclassification of clostridial cluster iii members into four novel genera in a new hungateiclostridiaceae fam. nov. Int. J. Syst. Evol. Microbiol. 68, 3197-3211 (2018).

26. Girbal, L., Croux, C., Vasconcelos, I. \& Soucaille, P. Regulation of metabolic shifts in Clostridium acetobutylicum ATCC 824. FEMS Microbiol. Rev. 17, 287-297 (1995).

575 27. Qi, F. et al. Improvement of butanol production in Clostridium acetobutylicum through enhancement of NAD(P)H availability. J. Ind. Microbiol. Biotechnol. 45, 993-1002 (2018).

28. Branduardi, P., Longo, V., Berterame, N. M., Rossi, G. \& Porro, D. A novel pathway to produce butanol and isobutanol in Saccharomyces cerevisiae. Biotechnol. Biofuels 6, 1

580 (2013).

29. Johnsen, U. \& Schönheit, P. Novel xylose dehydrogenase in the halophilic archaeon Haloarcula marismortui. J. Bacteriol. 186, 6198-6207 (2004).

30. Ravachol, J. et al. Mechanisms involved in xyloglucan catabolism by the cellulosomeproducing bacterium Ruminiclostridium cellulolyticum. Sci. Rep. 6, 1-17 (2016).

585 31. Macdonald, S. S., Blaukopf, M. \& Withers, S. G. N-acetylglucosaminidases from CAZy family $\mathrm{GH} 3$ are really glycoside phosphorylases, thereby explaining their use of histidine as an acid/Base catalyst in place of glutamic acid. J. Biol. Chem. 290, 4887-4895 (2015).

32. Jenney, F. E. \& Adams, M. W. W. Hydrogenases of the model hyperthermophiles. Ann. N. Y. Acad. Sci. 1125, 252-266 (2008).

590 33. Van Haaster, D. J., Silva, P. J., Hagedoorn, P. L., Jongejan, J. A. \& Hagen, W. R. Reinvestigation of the steady-state kinetics and physiological function of the soluble NiFehydrogenase I of Pyrococcus furiosus. J. Bacteriol. 190, 1584-1587 (2008).

34. Greening, C. et al. Genomic and metagenomic surveys of hydrogenase distribution indicate $\mathrm{H} 2$ is a widely utilised energy source for microbial growth and survival. ISME J. $595 \quad$ 10, 761-777 (2016).

35. Stetter, K. O. Hyperthermophiles in the history of life. Philos. Trans. R. Soc. B Biol. Sci. 361, 1837-1842 (2006).

36. Adam, P. S., Borrel, G. \& Gribaldo, S. An archaeal origin of the Wood-Ljungdahl H4MPT branch and the emergence of bacterial methylotrophy. Nat. Microbiol. 4, (2019). 
600 37. Collins, T., Gerday, C. \& Feller, G. Xylanases, xylanase families and extremophilic xylanases. FEMS Microbiol. Rev. 29, 3-23 (2005).

38. Schädel, C., Richter, A., Blöchl, A. \& Hoch, G. Hemicellulose concentration and composition in plant cell walls under extreme carbon source-sink imbalances. Physiol. Plant. 139, 241-255 (2010).

605 39. Wang, Y. et al. Environmental Conditions Constrain the Distribution and Diversity of Archaeal merA in Yellowstone National Park, Wyoming, U.S.A. Microb. Ecol. 62, 739 752 (2011).

40. Chen, S. et al. The Great Oxidation Event expanded the genetic repertoire of arsenic metabolism and cycling. (2020) doi:10.1073/pnas.2001063117.

610 41. Rogers, K. L. \& Schulte, M. D. Organic sulfur metabolisms in hydrothermal environments. Geobiology 10, 320-32 (2012).

42. Rabus, R., Venceslau, S. S., Lars, W., Wall, J. D. \& Pereira, I. A. C. A Post-Genomic View of the Ecophysiology, Catabolism and Biotechnological Relevance of SulphateReducing Prokaryotes. in Adv Microb Physiol vol. 66 55-321 (2015).

615 43. Nunes, C. I. P. et al. ArsC3 from Desulfovibrio alaskensis G20, a cation and sulfateindependent highly efficient arsenate reductase. J. Biol. Inorg. Chem. 19, 1277-1285 (2014).

44. Baker, B. J. et al. Genomic inference of the metabolism of cosmopolitan subsurface Archaea, Hadesarchaea. Nat. Microbiol. 1, (2016).

620 45. Seitz, K. W. et al. New Asgard archaea capable of anaerobic hydrocarbon cycling. Nat. Commun. 10, 1822 (2019).

46. Baker, B. J. et al. Diversity, ecology and evolution of Archaea. Nat. Microbiol. 29, (2020).

47. Hatzenpichler, R., Krukenberg, V., Spietz, R. L. \& Jay, Z. J. Next-generation physiology approaches to study microbiome function at single cell level. Nat. Rev. Microbiol. 18, 241-256 (2020).

48. Eren, A. M. et al. Anvi'o: an advanced analysis and visualization platform for 'omics data. PeerJ 3, e1319 (2015).

49. Kang, D. D., Froula, J., Egan, R. \& Wang, Z. MetaBAT, an efficient tool for accurately reconstructing single genomes from complex microbial communities. PeerJ 3, e1 165 (2015).

50. Dick, G. J. et al. Community-wide analysis of microbial genome sequence signatures. Genome Biol. 10, (2009).

51. Darling, A. E. et al. PhyloSift: phylogenetic analysis of genomes and metagenomes. PeerJ

52. Hyatt, D. et al. Prodigal: prokaryotic gene recognition and translation initiation site identification. BMC Bioinformatics 11, 119 (2010).

53. Aramaki, T. et al. KofamKOALA: KEGG Ortholog assignment based on profile HMM and adaptive score threshold. Bioinformatics 36, 2251-2252 (2020).

640 54. Jones, P. et al. InterProScan 5: Genome-scale protein function classification. Bioinformatics 30, 1236-1240 (2014).

55. Søndergaard, D., Pedersen, C. N. S. \& Greening, C. HydDB: A web tool for hydrogenase classification and analysis. Sci. Rep. 6, 1-8 (2016).

56. Zhang, H. et al. DbCAN2: A meta server for automated carbohydrate-active enzyme 645 annotation. Nucleic Acids Res. 46, W95-W101 (2018).

57. De Anda, V. et al. MEBS, a software platform to evaluate large (meta)genomic collections according to their metabolic machinery: Unraveling the sulfur cycle. Gigascience 6, 1-17 (2017).

58. Zhou, Z., Tran, P., Liu, Y., Kieft, K. \& Anantharaman, K. METABOLIC: A scalable high- 
650 throughput metabolic and biogeochemical functional trait profiler based on microbial genomes. bioRxiv 761643 (2019) doi:10.1101/761643.

59. Taboada, B., Estrada, K., Ciria, R. \& Merino, E. Operon-mapper: a web server for precise operon identification in bacterial and archaeal genomes. Bioinformatics 34, 4118-4120 (2018).

655 60. Lombard, V., Golaconda Ramulu, H., Drula, E., Coutinho, P. M. \& Henrissat, B. The carbohydrate-active enzymes database (CAZy) in 2013. Nucleic Acids Res. 42, 490-495 (2014).

61. Yu, N. Y. et al. PSORTb 3.0: Improved protein subcellular localization prediction with refined localization subcategories and predictive capabilities for all prokaryotes.

660 Bioinformatics 26, 1608-1615 (2010).

62. Boyd, J. A., Woodcroft, B. J. \& Tyson, G. W. GraftM : a tool for scalable, phylogenetically informed classification of genes within metagenomes. 46, (2018).

63. Hua, Z. S. et al. Insights into the ecological roles and evolution of methyl-coenzyme M reductase-containing hot spring Archaea. Nat. Commun. 10, 1-11 (2019).

665 64. Chaumeil, P., Mussig, A. J., Parks, D. H. \& Hugenholtz, P. Genome analysis GTDB-Tk : a toolkit to classify genomes with the Genome Taxonomy Database. 36, 1925-1927 (2020). 


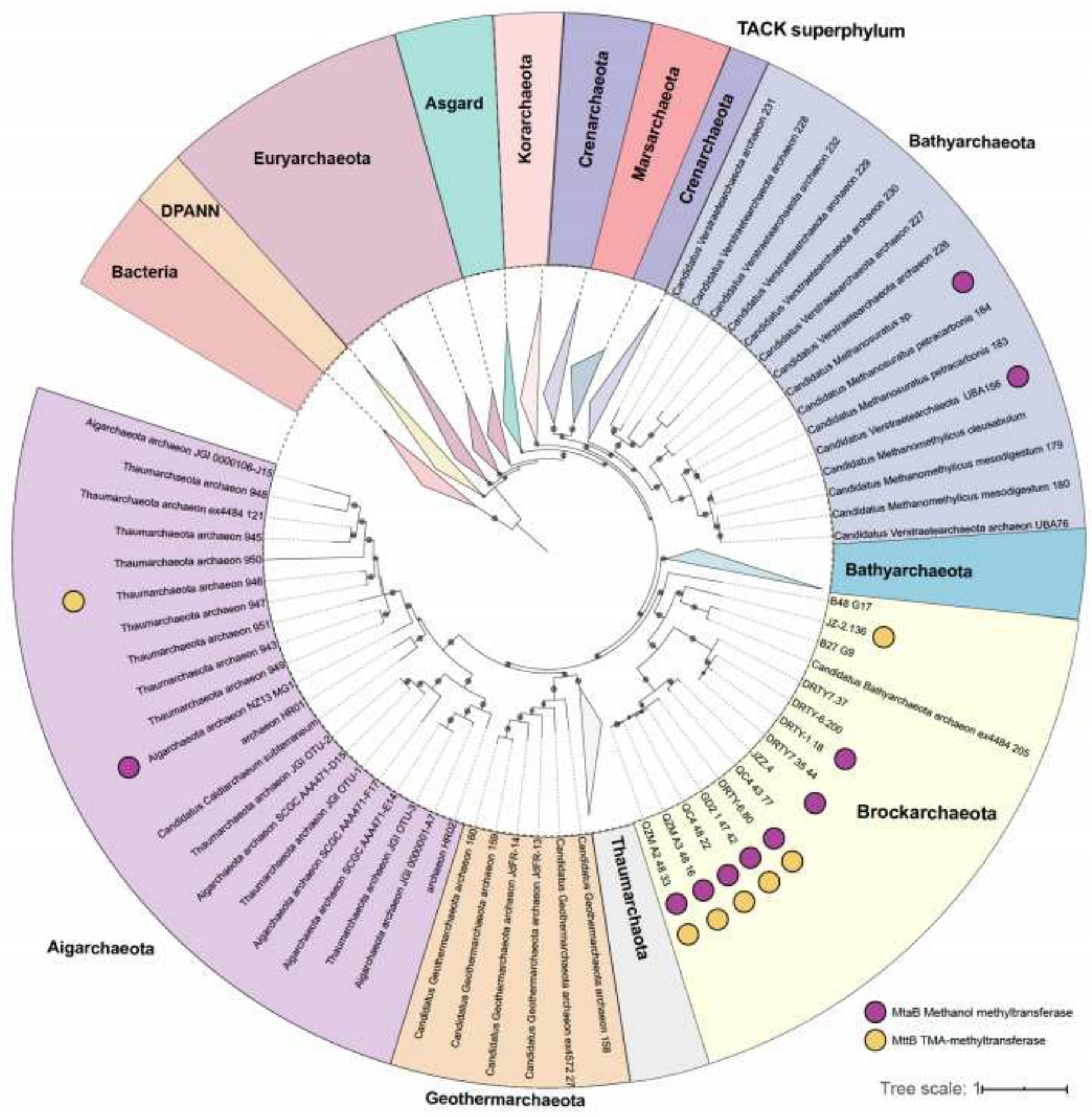

Figure 1

. Comparison of phylogeny and distribution of methyltransferase system in Brockarchaeota and other members of TACK superphylum. Phylogeny generated using iqtreev1.6.1 using a concatenation of 37 conserved single-copy protein-coding genes described in ref14. Bootstrap values were calculated using non-parametric bootstrapping with 100 replicates (represented by gray circles, only bootstrap $>70$ are 
shown). The presence methanol methyltransferase MtaB (PF12176) and trimethylamine methyltransferase MttB (PF06253) are shown in the outer circles. The annotation was conducted with MEBS15 details can found in Supporting Table 5.

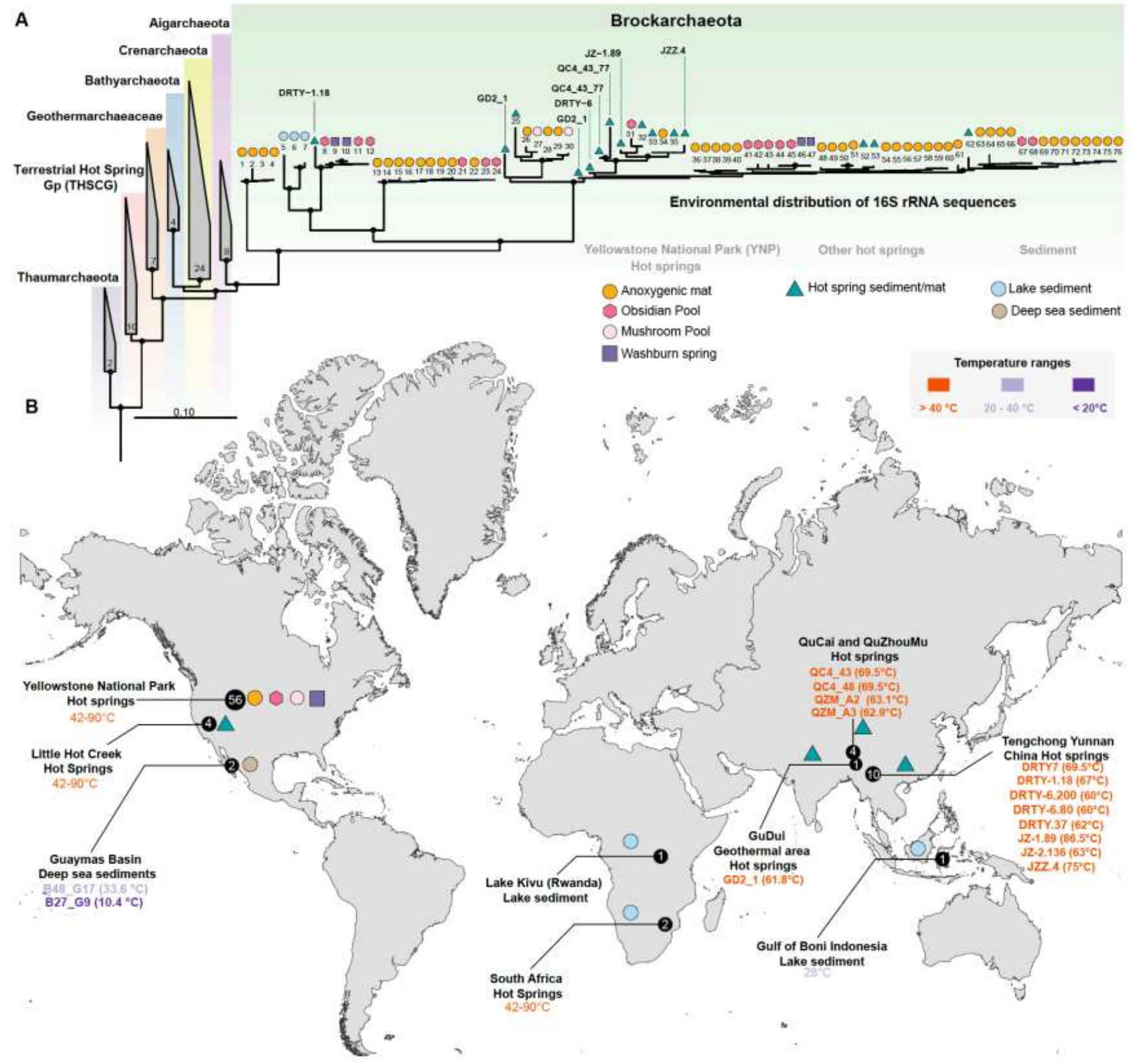

Figure 2

Location of samples from which Brockarchaeota genomes and 16S rRNA gene sequences have been recovered. (A) 16S rRNA gene tree of sequences derived from metagenomic and rRNA-based diversity surveys (NCBI accessions EU924237, KX213943, and KX213897). The eight complete 16S rRNA gene sequences of Brockarchaeota genomes described in this study are shown in their respective names. Black circles in the tree represent 100 bootstrap values using RAxML with 100 replicates. Environmental information of each sequence is shown was obtained from Integrated Microbial Genomes and 
Microbiomes database. The number of the sequences and the corresponding metadata are described in Supporting Table 4. (B) Geographic localization from which Brockarchaeota sequences where obtained. The size of the circle corresponds to the total number of Brockarchaeota-related sequences in each geographic location. The specific MAGs obtained in this study are shown in the map according to their temperature range. Note: The designations employed and the presentation of the material on this map do not imply the expression of any opinion whatsoever on the part of Research Square concerning the legal status of any country, territory, city or area or of its authorities, or concerning the delimitation of its frontiers or boundaries. This map has been provided by the authors.

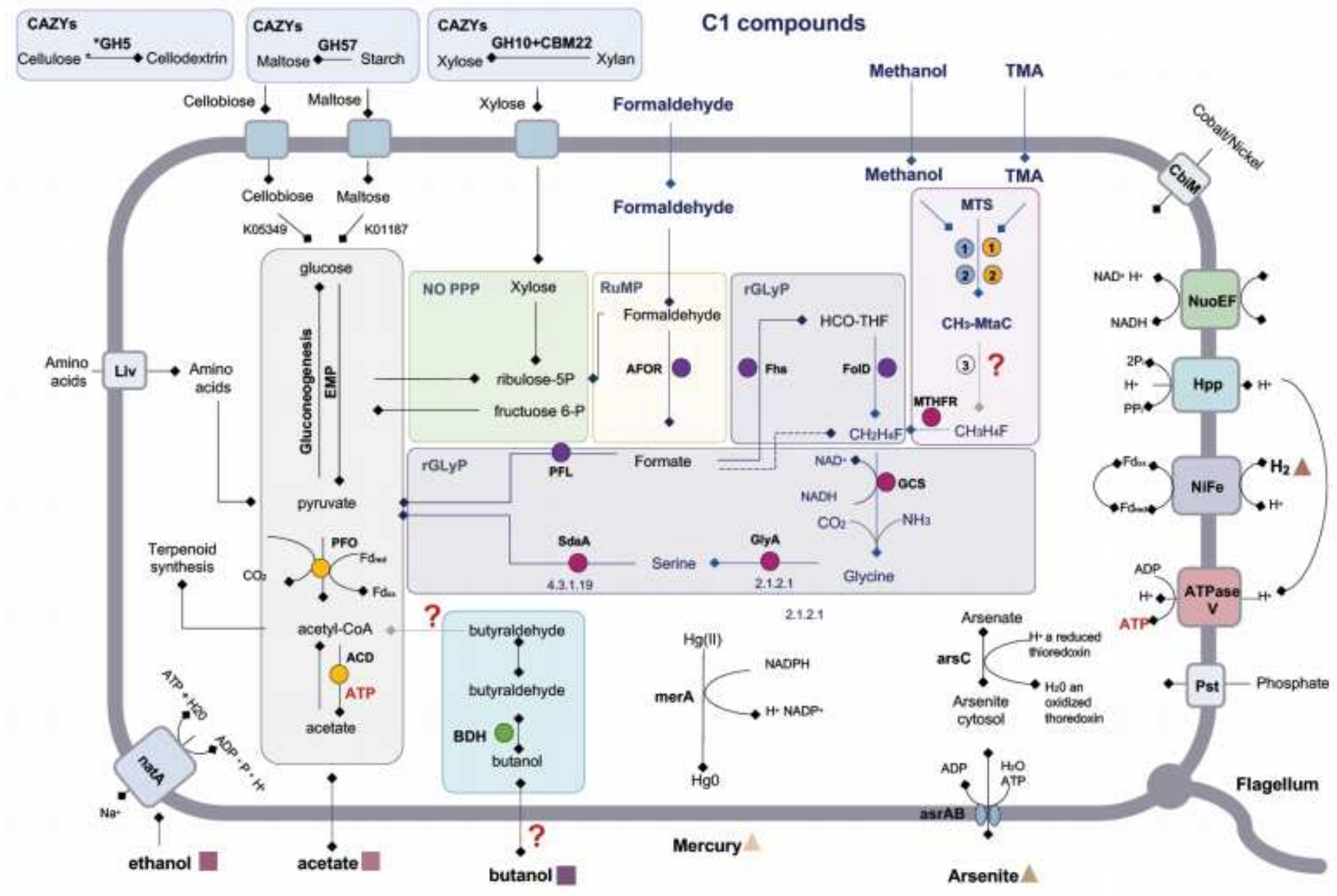

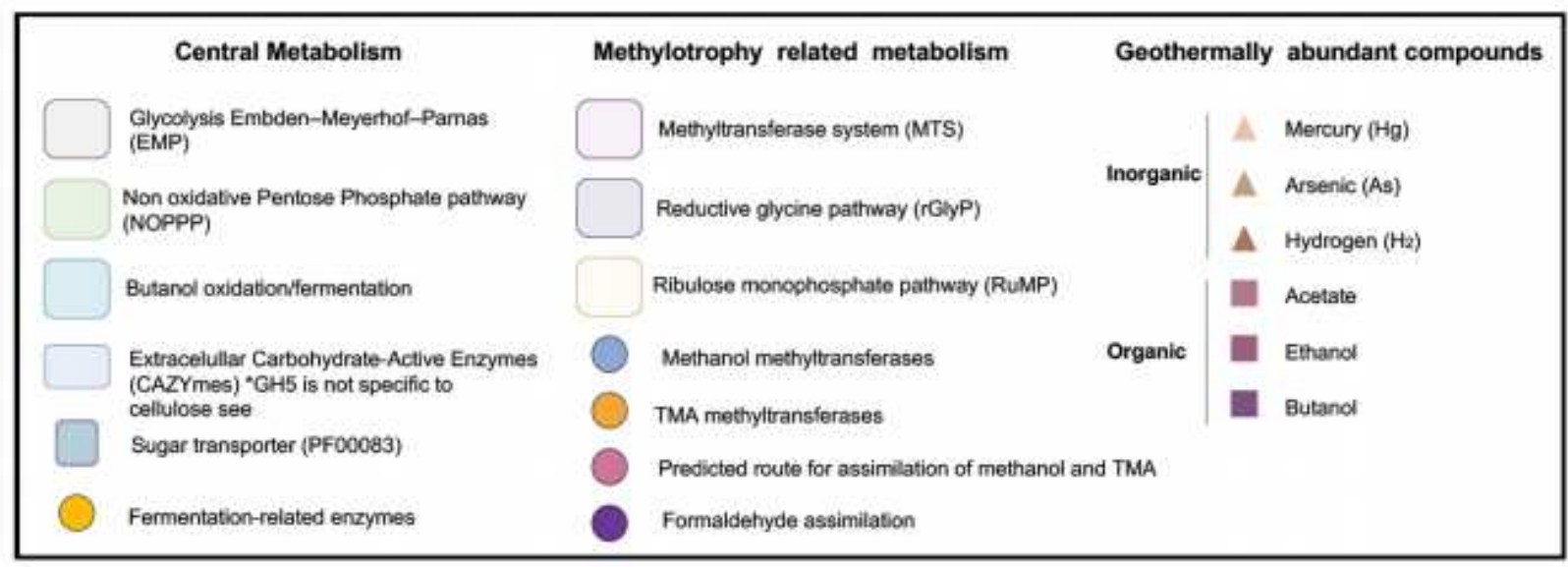


Overview of potential metabolic capabilities of Brockarchaeota phylum. Brockarchaota is predicted to produce ATP by substrate-level phosphorylation by fermentation of complex organic compounds, pyruvate and acetate as carbon and energy sources via glycolysis (EMP) by the concerted action of PFO and $A C D$, that might represent the major energy-conserving reaction in this lineage. Formaldehyde can be fixed and detoxified via the ribulose monophosphate (RuMP) pathway and enter to central metabolism or be oxidized to formate by AFOR which can enter to the rGLyP pathway via FhS and FolD action C1 compounds such as methanol and TMA can be assimilated via MT system and MTHFR, generating glycine, and following a linear route to central metabolism through rGLyP by subsequent conversion of serine and pyruvate. Geothermally abundant compounds are showed in colored circles Each shaded pathway can be seen in more detail in Supporting Discussion. Dashed arrow indicates that spontaneous condensation of formaldehyde with THF occur but is a very minor contribution to assimilation fluxes according to ref36. For full names and copy numbers of the genes in number see Supporting Discussion. Abbreviations. Pathways:Embden-Meyerhof-Parnas (EMP), Non-Oxidative Pentoses Phosphate Pathway (NOPPP), ribulose monophosphate (RuMP), reductive glycine pathway (rGlyP). Enzymes: pyruvate ferredoxin oxidoreductase (PFO), and acetate-CoA ligase (ADP-forming) (ACD), pyruvate formate lyase (PFL), tungsten-dependent aldehyde ferredoxin oxidoreductase (AFOR), tetrahydrofolate (THF) ligase (FhS), methenyl-THF cyclohydrolase/methylene-THF dehydrogenase (FolD), methylene-THF reductase MTHFR (MetFmethyltransferase system (MT), glyA, serine hydroxymethyltransferase (GlyA), serine deaminase (SdaA), glycine-cleavage system (GCS). Compounds: Single carbon compounds (C1), trimethylamine (TMA). The three main components of the methyltransferase system are explained in the main text. 


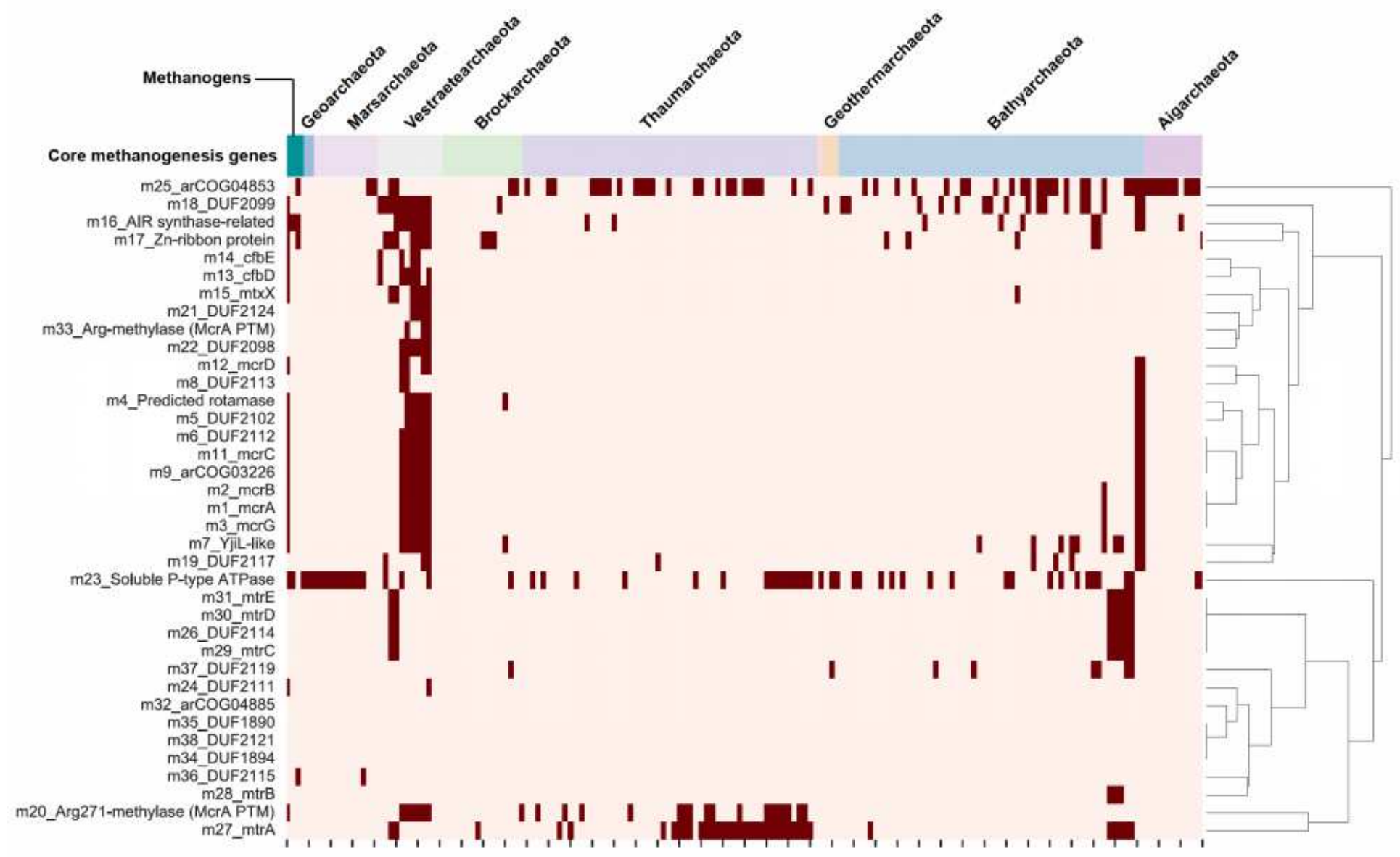

Figure 4

Presence and absence profile of common core marker genes specific to methanogenesis across the TACK superphylum. Genes include those associated to methanogenesis, anaerobic methanotrophic and shortchain alkane-oxidizing archaea described in Greening et al. 201634 (see Supporting Table 6 for specific details of the genomes used in this study and the specific marker genes named with $\mathrm{m}$ followed with a number in the figure). For comparison purposes the following known methanogenic archaea were included: Candidatus Methanoplasma termitum that lacks the entire pathway for $\mathrm{CO} 2$ reduction to methyl coenzyme $M$ and produces methane by hydrogen-dependent reduction of methanol or methylamine (Methanomassiliicoccales), Methanosphaera stadtmanae (Methanobacteriales) that can generate methane only by the reduction of methanol with $\mathrm{H} 2$ and is dependent on acetate as a carbon source, and Methanosarcina acetivorans (Methanosarcinales). 


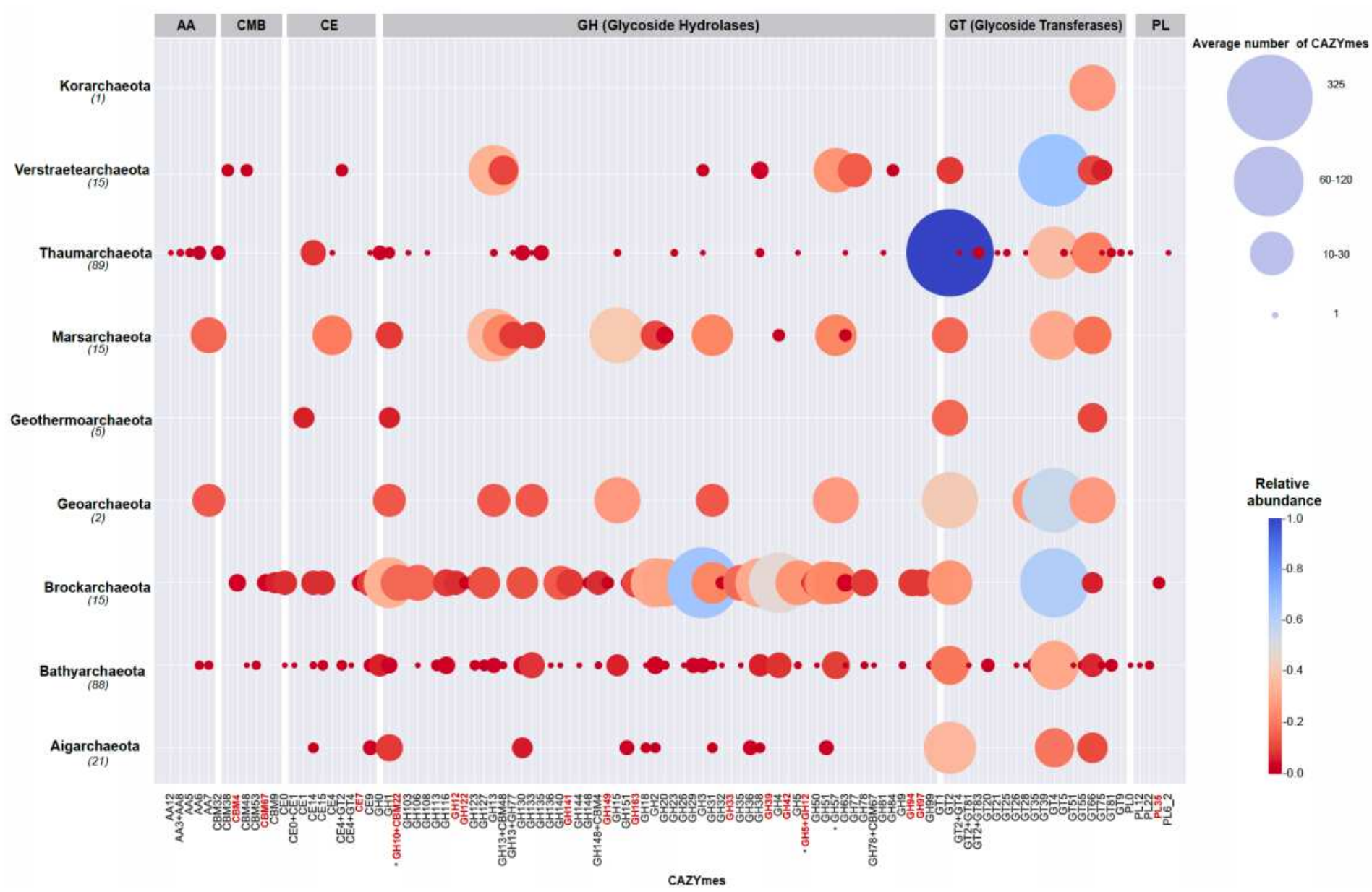

\section{Figure 5}

Annotated carbohydrate-active enzymes (CAZymes) encoded by the total number of genomes belonging to the TACK superphylum including Brockarchaeota. The total number of CAZymes per phylum was normalized by the total number of genomes described for each phylum shown in parenthesis. Auxiliary activities (AA), carbohydrate-binding module (CBM), carbohydrate esterases (CE), glycoside-hydrolases $(\mathrm{GH})$, glycoside transferases (GT), polysaccharide lyases (PL). Unique Brockarchaeota CAZymes are shown in red. Extracellular CAZYmes in Brockarchaeota are shown in asterisks described in Supporting Table 8 sheet 3 . 


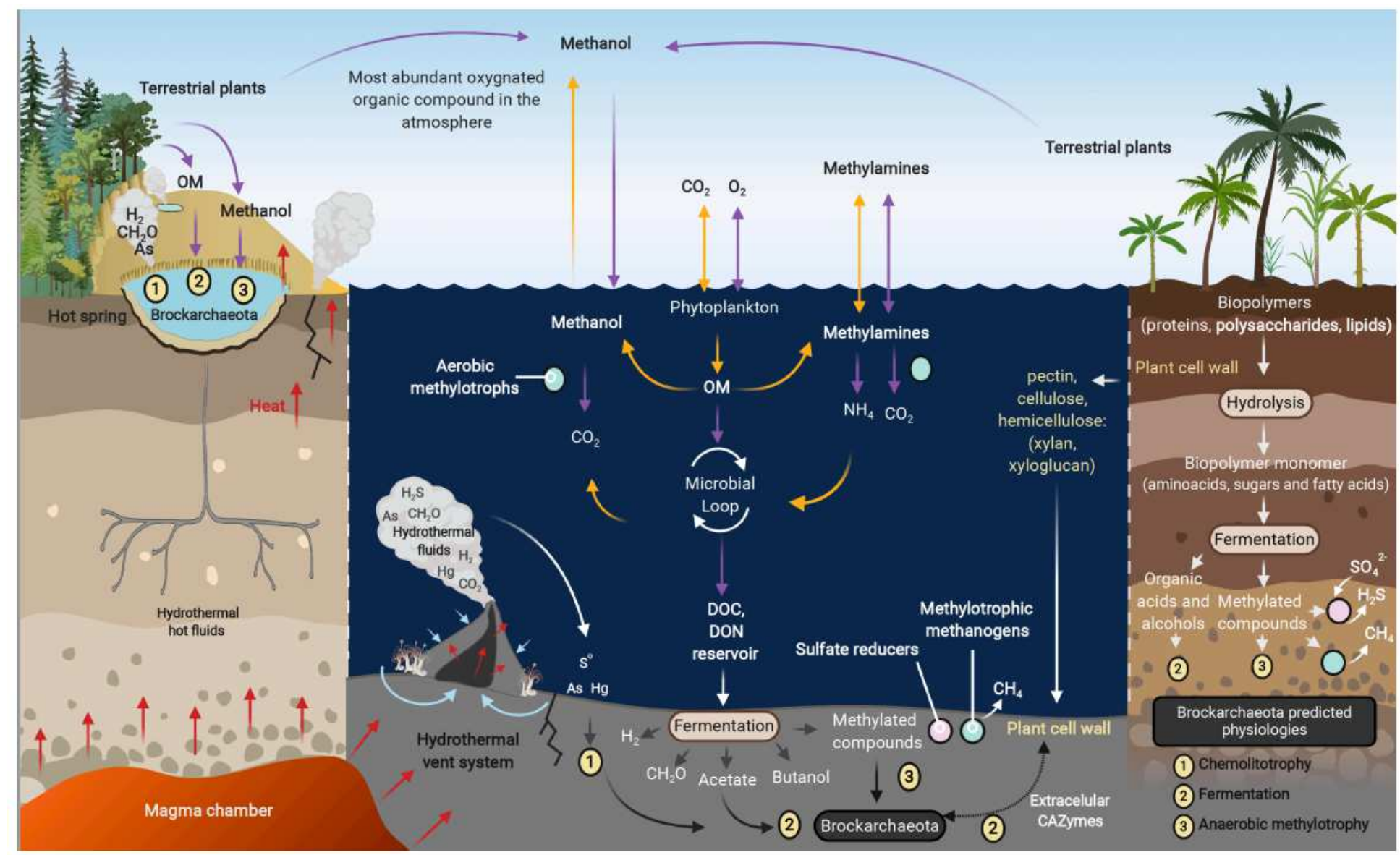

\section{Figure 6}

The role of Brockarchaeota in the anaerobic carbon cycle. Single carbon (C1)-methylated compounds, such as methanol or methylamines, are utilized biologically as carbon and energy sources in the ocean and deep-sea sediments resulting in a considerable carbon reservoir. The biodegradation of organic carbon in the water column and subsurface is a source of C1-methylated compounds. The utilization of methyl compounds as precursors in methane synthesis is confined to a small group of methylotrophic methanogens (i.e Verstraetearchaeota). The only described anaerobic methylotrophs include members of methanogenic archaea, acetogenic bacteria, and sulfate-reducing bacteria. These organisms compete for $\mathrm{C} 1$ compounds geochemically produced in anoxic settings. Brockarchaeota may recycle $\mathrm{C} 1$ in anoxic environments without methane formation and may be sequestered in deep sea sediments and hot springs. Orange and purple arrows represent sources and sinks, respectively. Organic Matter (OM) includes dissolved and particulate organic matter feeding the microbial loop (Adapted from Evans et al., 2019 and Zhuang et al., 2018).

\section{Supplementary Files}

This is a list of supplementary files associated with this preprint. Click to download.

- Description.pdf 